\title{
A New Chiral Rh(II) Catalyst for Enantioselective [2+1]-Cycloaddition. Mechanistic Implications and Applications
}

\author{
Yan Lou, Manabu Horikawa, Robin A. Kloster, Natalie A. Hawryluk and E. J. Corey* \\ Department of Chemistry and Chemical Biology \\ Harvard University, Cambridge, Massachusetts, 02138, USA
}

\section{Supplementary Material}

Part 1. Preparation of Catalysts $\mathbf{1 , 2}$ and $\mathbf{1 0}$

S 2

Part 2. Cyclopropenation Reactions with Ethyl Diazoacetate Catalyzed by $1 \quad$ S 7

Part 3. Cyclopropanation and Other Reactions Catalyzed by $1 \quad$ S 11

Part 4. Transformations of Ethyl (1S)-2-n-Pentyl-2-cyclopropene-carboxylate $\quad$ S 15

Part 5. Synthesis of $(9 R, 10 S)$-Dihydrosterculic Acid (12) S 20

Part 6. ORTEP Presentations of Catalysts 1, 2 and $\mathbf{1 0} \quad$ S 25

Materials and Methods. Unless stated otherwise, reactions were performed in flamedried glassware under a positive pressure of nitrogen using freshly distilled solvent. Tetrahydrofuran (THF) and diethyl ether were freshly distilled from sodium/ benzophenone before use. Dichloromethane was freshly distilled from $\mathrm{CaH}_{2}$ before use. Toluene was distilled from sodium. Thin-layer chromatography (TLC) was performed using E. Merck silica gel $60 \mathrm{~F}_{254}$ pre-coated plates $(0.25 \mathrm{~mm})$. Merck $\mathrm{CN} \mathrm{F}_{254}$ plates were used in the preparation of catalyst $\mathbf{1}$. Preparative thin-layer chromatography separations were carried out on $0.50 \mathrm{~mm}$ E. Merck silica gel plates (60F-254). Column 
chromatography was performed using Bakerbond silica gel (40 $\mu \mathrm{m}$ particle size).

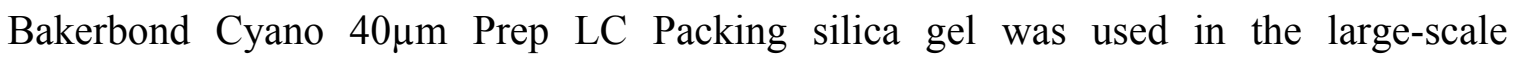
purification of catalyst 1. NMR spectra were recorded on Varian Innova-500, or Mercury-400 instruments using residual undeuterated solvent as an internal standard. IR spectra were recorded on an Avatar 360 FT-IR spectrometer. Optical rotation was measured using a Perkin-Elmer 241 Polarimeter. High-resolution mass spectral analyses were performed at Harvard University Mass Spectrometry Center. Analytical high performance liquid chromatography (HPLC) was performed on an Isco 2350 Series or a Waters 626 HPLC using the indicated chiral columns. Gas chromatography (GC) analyses were performed on a Hewlett-Packard 6850 Series GC System equipped with flame ionization detector using a J \& W Scientific Cyclosil-B column (30 x $0.25 \mathrm{~mm})$ or an Alltech Chiraldex $\gamma$-TA column $(30 \times 0.25 \mathrm{~mm})$. Commercial grade reagents and solvents were used without further purification unless otherwise stated. All isolated compounds described herein were judged to be of $\geq 95 \%$ purity as determined by ${ }^{1} \mathrm{H}$ and ${ }^{13} \mathrm{C}$ NMR spectroscopy.

\section{Part 1. Preparation of Catalysts 1, 2 and 10} $(4 R, 5 R)$-Diphenyl-imidazolidin-2-one ${ }^{1}$

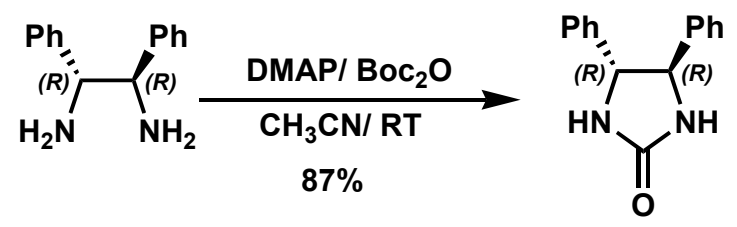

$(1 R, 2 R)$-Diphenylethanediamine $(0.537 \mathrm{~g}, 2.53 \mathrm{mmol})$ and DMAP $(0.309 \mathrm{~g}, 2.78$ mmol) were dissolved in $\mathrm{CH}_{3} \mathrm{CN}(40 \mathrm{~mL})$. Boc $2 \mathrm{O}(0.607 \mathrm{~g}, 2.78 \mathrm{mmol})$ was added in one portion and the reaction mixture was stirred at room temperature overnight. After 
removal of solvent by rotary evaporation, the residue was purified by column chromatography $\left(1: 1 \mathrm{CH}_{2} \mathrm{Cl}_{2}\right.$-EtOAc) to provide $0.53 \mathrm{~g}(87 \%)$ of the known cyclic urea ${ }^{1}$ as white solid: $[\alpha]^{23}{ }_{\mathrm{D}}=+96^{\circ}\left(c\right.$ 1.40, $\left.\mathrm{CHCl}_{3}\right) ;{ }^{1} \mathrm{H}$ NMR $\left(\mathrm{CDCl}_{3}, 400 \mathrm{MHz}\right): \delta 7.37(\mathrm{~m}$, $6 \mathrm{H}), 7.27(\mathrm{~d}, J=7.5 \mathrm{~Hz}, 4 \mathrm{H}), 4.90(\mathrm{~s}, 2 \mathrm{H}), 4.60(\mathrm{~s}, 2 \mathrm{H}) \mathrm{ppm}$.

\section{(4R,5R)-Diphenyl-1-trifluoromethanesulfonyl-imidazolidin-2-one}

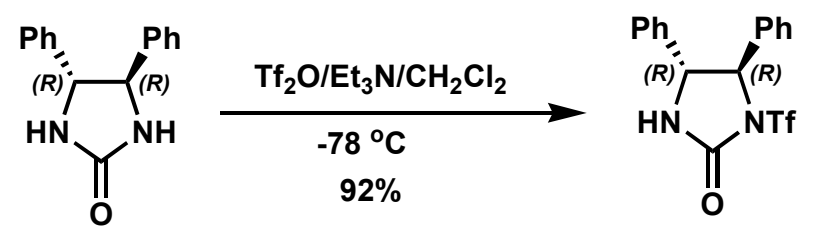

A solution of $(4 R, 5 R)$-diphenyl-imidazolidin-2-one $(0.522 \mathrm{~g}, 2.19 \mathrm{mmol})$ and $\mathrm{Et}_{3} \mathrm{~N}$ (1.22 mL, $8.76 \mathrm{mmol})$ in $\mathrm{CH}_{2} \mathrm{Cl}_{2}(10 \mathrm{~mL})$ was cooled to $-78{ }^{\circ} \mathrm{C}$. Triflic anhydride $(0.85$ $\mathrm{mL}, 5.0 \mathrm{mmol}$ ) was added dropwise via syringe. The resulting solution was stirred at -78 ${ }^{\circ} \mathrm{C}$ for $45 \mathrm{~min}$ and 1:1 $\mathrm{H}_{2} \mathrm{O}-\mathrm{MeOH}(5 \mathrm{~mL})$ was added. The reaction mixture was warmed to room temperature, maintained for 30 minutes, and then diluted with $\mathrm{Et}_{2} \mathrm{O}(300 \mathrm{~mL})$. The organic layer was washed with $1 \mathrm{~N} \mathrm{HCl}(50 \mathrm{~mL})$, brine $(50 \mathrm{~mL})$, dried, filtered and then concentrated in vacuo. Purification of this residue by flash chromatography $\left(\mathrm{SiO}_{2}\right.$, $\left.100 \% \mathrm{CH}_{2} \mathrm{Cl}_{2}\right)$ gave the mono triflamide $(0.62 \mathrm{~g}, 92 \%)$ as a white solid, mp $132{ }^{\circ} \mathrm{C}$; $[\alpha]^{23}{ }_{\mathrm{D}}=+86^{\mathrm{o}}\left(\mathrm{c} 3.5, \mathrm{CHCl}_{3}\right) ;{ }^{1} \mathrm{H} \mathrm{NMR}\left(\mathrm{CDCl}_{3}, 400 \mathrm{MHz}\right): \delta 7.49-7.36(\mathrm{~m}, 8 \mathrm{H}), 7.25(\mathrm{dd}$, $J=2.0 \mathrm{~Hz}, 8.0 \mathrm{~Hz}, 2 \mathrm{H}), 5.89$ (br s, $1 \mathrm{H}), 5.01(\mathrm{~d}, J=3.5 \mathrm{~Hz}, 1 \mathrm{H}), 4.77 \mathrm{ppm}(\mathrm{d}, J=3.5$ $\mathrm{Hz}, 1 \mathrm{H}) ;{ }^{13} \mathrm{C} \mathrm{NMR}\left(\mathrm{CDCl}_{3}, 400 \mathrm{MHz}\right): \delta 153.5,139.3,138.8,129.7,129.6,129.6,129.4$, 126.4, 125.5, 119.0 (q, $J=321 \mathrm{~Hz}$ ), 69.9, 62.4 ppm; FTIR (film): 3314, 1758, 1457 , 1408, 1378, 1221, 1208, 1148, 1102, 1079, 1058, $1029 \mathrm{~cm}^{-1}$; HRMS $\left(\mathrm{ESI}^{+}\right)$calcd for $\mathrm{C}_{16} \mathrm{H}_{13} \mathrm{~N}_{2} \mathrm{O}_{3} \mathrm{~F}_{3} \mathrm{~S}(\mathrm{M}+\mathrm{H})^{+}$371.0677, found 371.0692 .

Procedure for the preparation of $(R, R)-\mathrm{Rh}_{2}(\mathrm{OAc})(\mathrm{DPTI})_{3}(1)$ 


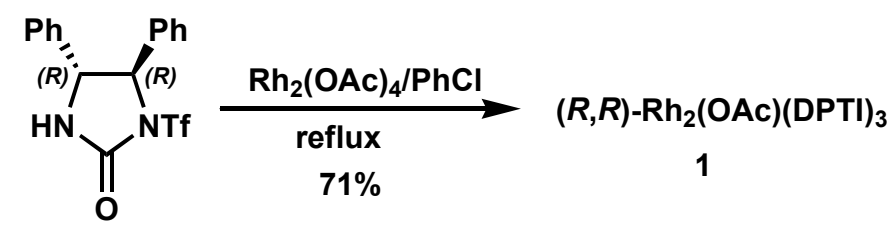

A flame-dried $5 \mathrm{~mL}$ round bottom flask was fitted with a Dean-Stark apparatus that was charged with a 5:1 mixture of anhydrous $\mathrm{Na}_{2} \mathrm{CO}_{3}$ and $4 \AA$ molecular sieves (powder) covered by a layer of cotton to prevent the solid mixture from entering the reaction flask. A mixture of $\mathrm{Rh}_{2}(\mathrm{OAc})_{4} \quad(44 \mathrm{mg}, 0.099 \mathrm{mmol})$ and (4R,5R)-diphenyl-1trifluoromethanesulfonyl-imidazolidin-2-one $(115 \mathrm{mg}, 0.310 \mathrm{mmol})$ in chlorobenzene (3 $\mathrm{mL})^{\mathrm{a}}$ was transferred into the $5 \mathrm{~mL}$ flask and heated at reflux with vigorous stirring under nitrogen in the Dean-Stark setup. Reflux was continued until all insoluble $\mathrm{Rh}_{2}(\mathrm{OAc})_{4}$ was consumed and TLC analysis $\left(10: 1 \mathrm{C}_{6} \mathrm{H}_{6}-\mathrm{CH}_{3} \mathrm{CN}\right)$ of the deep blue solution indicated that 1 had formed as the predominant product (ca. $20 \mathrm{~h}){ }^{\mathrm{b}}$ After cooling, the reaction mixture was purified by silica gel chromatography $\left(100 \% \mathrm{PhCH}_{3} \rightarrow 5 \% \mathrm{CH}_{3} \mathrm{CN}\right.$ in $\left.\mathrm{PhCH}_{3}\right)$ to give a purple solid, which contained a small impurity having a slightly higher $\mathrm{R}_{\mathrm{f}}$ than $\mathbf{1}^{\mathrm{c}}$ This slightly impure product was further purified by preparative thin-layer chromatography with $\mathrm{CH}_{2} \mathrm{Cl}_{2}$ to afford pure 1 as purple solid (97 mg, 71\%): TLC (10:1 $\mathrm{C}_{6} \mathrm{H}_{6}-\mathrm{CH}_{3} \mathrm{CN}, 0.25 \mathrm{~mm}$ E. Merck silica gel $60 \mathrm{~F}_{254}$ precoated plate $), \mathrm{R}_{\mathrm{f}}=0.4 ;(20: 1$ EtOAc-MeOH, Merck CN F 254 plate $)^{\mathrm{d}}, \mathrm{R}_{\mathrm{f}}=0.3 ; \mathrm{mp}>250^{\circ} \mathrm{C} ;[\alpha]^{23}{ }_{\mathrm{D}}=+185^{\circ}(c 0.043$, $\left.\mathrm{CHCl}_{3}\right) ;{ }^{1} \mathrm{H} \mathrm{NMR}\left(\mathrm{CDCl}_{3}, 500 \mathrm{MHz}\right): \delta 7.62(\mathrm{t}, J=7.5 \mathrm{~Hz}, 2 \mathrm{H}), 7.54(\mathrm{t}, J=7.5 \mathrm{~Hz}, 1 \mathrm{H})$, $7.48(\mathrm{t}, J=7.5 \mathrm{~Hz}, 2 \mathrm{H}), 7.41-7.16(\mathrm{~m}, 17 \mathrm{H}), 6.99(\mathrm{~m}, 2 \mathrm{H}), 6.92(\mathrm{dd}, J=7.5 \mathrm{~Hz}, 15 \mathrm{~Hz}$, 4H), $6.74(\mathrm{t}, J=7.5 \mathrm{~Hz}, 2 \mathrm{H}), 4.99(\mathrm{~d}, J=2.5 \mathrm{~Hz}, 1 \mathrm{H}), 4.92(\mathrm{~d}, J=2.5 \mathrm{~Hz}, 1 \mathrm{H}), 4.79(\mathrm{~d}, J$ $=2.5 \mathrm{~Hz}, 1 \mathrm{H}), 4.56(\mathrm{~d}, J=2.5 \mathrm{~Hz}, 1 \mathrm{H}), 4.08(\mathrm{~d}, J=2.5 \mathrm{~Hz}, 1 \mathrm{H}), 3.86(\mathrm{~d}, J=2.5 \mathrm{~Hz}, 1 \mathrm{H})$ ppm; ${ }^{19} \mathrm{~F} \mathrm{NMR}\left(\mathrm{CDCl}_{3}, 400 \mathrm{MHz}\right): \Phi-74.7$ (s, 1F), -75.0 (s, 1F), -75.1 (s, 1F) ppm; FTIR (film): 1667, 1402, 1227, 1198, 1065, $802 \mathrm{~cm}^{-1}$; MS (FAB) calcd for 
$\mathrm{C}_{50} \mathrm{H}_{39} \mathrm{~F}_{9} \mathrm{~N}_{6} \mathrm{O}_{11} \mathrm{Rh}_{2} \mathrm{~S}_{3}(\mathrm{M})^{+} 1372$, found 1372 . The structure of $\mathbf{1}$ was determined by X-ray diffraction method.

Note:

a. Using more dilute conditions resulted in slower reaction and a lower yield of the desired product 1 .

b. A significant amount of a purple dirhodium compound that has slightly higher $R_{f}$ was formed more rapidly than $\mathbf{1}$, and is possibly a kinetically formed isomer of $\mathbf{1}$. Upon longer refluxing in chlorobenzene, this product was gradually converted into $\mathbf{1}$.

c. This minor impurity is the more rapidly formed "kinetic" isomer of $\mathrm{Rh}_{2}(\mathrm{OAc})(\mathrm{DPTI})_{3}$.

d. Alternatively, for large-scale purification, the higher $R_{f}$ impurity can be removed by

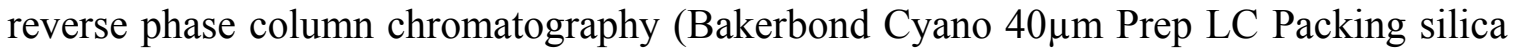
gel) with EtOAc as the eluent.

Procedure for the preparation of $(R, R)-\mathrm{Rh}_{2}(\mathrm{DPTI})_{4}(2)$

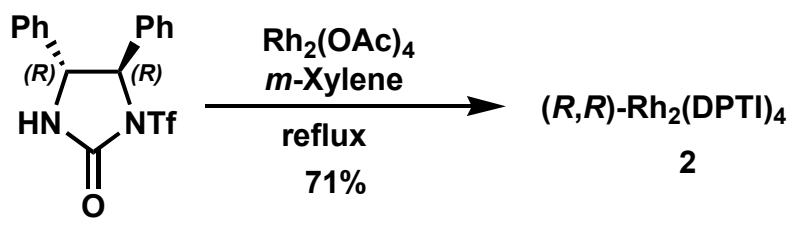

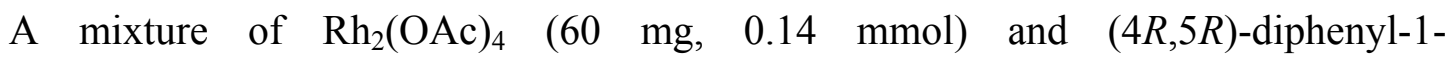
trifluoromethanesulfonyl-imidazolidin-2-one $(527 \mathrm{mg}, 1.72 \mathrm{mmol})$ in $m$-xylene $(5 \mathrm{~mL})$ was heated at reflux in a $10 \mathrm{~mL}$ round-bottom flask. The flask was equipped with a 10 $\mathrm{mL}$ pressure equalizing addition funnel, which contained $3 \mathrm{~g}$ of 5:1 mixture of $\mathrm{CaH}_{2} /$ Celite 545. The entire apparatus was wrapped in glass wool and aluminum foil to ensure a reflux rate of about $1 \mathrm{drop} / \mathrm{min}$ at the condenser. The deep blue mixture was heated for a total of $48 \mathrm{~h}$, ensuring that the solvent volume in the $10 \mathrm{~mL}$ flask was kept at 2-3 $\mathrm{mL}$. After TLC $\left(2 \% \mathrm{CH}_{3} \mathrm{CN}\right.$ in $\left.\mathrm{C}_{6} \mathrm{H}_{6}\right)$ indicated that the product formed was 
predominant, the mixture was cooled and concentrated in vacuo. Purification by silica gel chromatography (20:1 $\left.\mathrm{C}_{6} \mathrm{H}_{6}-\mathrm{CH}_{3} \mathrm{CN}\right)$ gave $115 \mathrm{mg}(49 \%)$ of $\mathrm{Rh}_{2}(\mathrm{DPTI})_{4}(2)$ as a purple solid: TLC $\left(20: 1 \mathrm{C}_{6} \mathrm{H}_{6}-\mathrm{CH}_{3} \mathrm{CN}\right), \mathrm{R}_{\mathrm{f}}=0.5 ;[\alpha]^{23}{ }_{\mathrm{D}}=+275^{\circ}\left(c 0.04, \mathrm{CHCl}_{3}\right) ;{ }^{1} \mathrm{H}$ NMR $(400$ $\left.\mathrm{MHz}, \mathrm{CDCl}_{3}\right): \delta 7.45-6.90(\mathrm{~m}, 30 \mathrm{H}), 6.8(\mathrm{~m}, 4 \mathrm{H}), 6.62(\mathrm{t}, J=7.2 \mathrm{~Hz}, 2 \mathrm{H}), 6.54(\mathrm{t}, J=7.2$ $\mathrm{Hz}, 2 \mathrm{H}), 6.45(\mathrm{~d}, J=7.2 \mathrm{~Hz}, 2 \mathrm{H}), 5.41(\mathrm{~d}, J=8.5 \mathrm{~Hz}, 1 \mathrm{H}), 5.17(\mathrm{~d}, J=1.5 \mathrm{~Hz}, 1 \mathrm{H}), 5.11$ $(\mathrm{d}, J=8.0 \mathrm{~Hz}, 1 \mathrm{H}), 4.97(\mathrm{~d}, J=5.0 \mathrm{~Hz}, 1 \mathrm{H}), 4.91(\mathrm{~d}, J=8.5 \mathrm{~Hz}, 1 \mathrm{H}), 4.87(\mathrm{~d}, J=8.0 \mathrm{~Hz}$, $1 \mathrm{H}), 3.27(\mathrm{~d}, J=4.5 \mathrm{~Hz}, 1 \mathrm{H}), 3.00(\mathrm{br} \mathrm{s}, 1 \mathrm{H}) \mathrm{ppm}$; MS (FAB) calcd for $\mathrm{C}_{64} \mathrm{H}_{48} \mathrm{~F}_{12} \mathrm{~N}_{6} \mathrm{O}_{12} \mathrm{Rh}_{2} \mathrm{~S}_{4}(\mathrm{M})^{+}$1681, found 1681 . The structure of 2 was determined by Xray diffraction method.

Procedure for the preparation of $(M)-(R, R)-c i s-(\mathrm{N}, \mathrm{O})-\mathrm{Rh}_{2}(\mathrm{Piv})_{2}(\mathrm{DPTI})_{2}(10)$

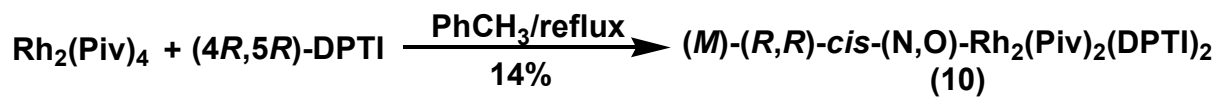

A mixture of dirhodium(II) tetrapivalate $(51 \mathrm{mg}, 0.084 \mathrm{mmol})$ and $(4 R, 5 R)$-diphenyl1-trifluoromethanesulfonyl-imidazolidin-2-one $(124 \mathrm{mg}, 0.334 \mathrm{mmol})$ in toluene $(1 \mathrm{~mL})$ was heated at reflux for approximately $36 \mathrm{~h}$. After cooling and concentration, the reaction mixture was purified with preparative thin-layer chromatography $\left(20: 1 \mathrm{C}_{6} \mathrm{H}_{6}-\mathrm{CH}_{3} \mathrm{CN}\right)$ to afford $\mathbf{1 0}$ as blue solid (14 $\mathrm{mg}, 14 \%)$. The yield of this preparation of $\mathbf{1 0}$ has not been optimized. TLC (20:1 $\left.\mathrm{C}_{6} \mathrm{H}_{6}-\mathrm{CH}_{3} \mathrm{CN}\right), \mathrm{R}_{\mathrm{f}}=0.4 ;{ }^{1} \mathrm{H} \mathrm{NMR}\left(\mathrm{CDCl}_{3}, 400 \mathrm{MHz}\right): \delta$ 7.6-7.1 (m, 20H), $4.92(\mathrm{~d}, J=5.0 \mathrm{~Hz}, 2 \mathrm{H}), 4.59(\mathrm{~d}, J=5.0 \mathrm{~Hz}, 2 \mathrm{H})$, and $1.08(\mathrm{~s}, 18 \mathrm{H}) \mathrm{ppm} ;{ }^{19} \mathrm{~F}$ NMR ( $\left.\mathrm{CDCl}_{3}, 400 \mathrm{MHz}\right)$ : $\Phi$-74.6 (s) ppm. The structure of $\mathbf{1 0}$ was determined by X-ray diffraction method. 


\section{Part 2. Cyclopropenation Reactions with Ethyl Diazoacetate Catalyzed by 1}

General procedure for the cyclopropenation reactions.

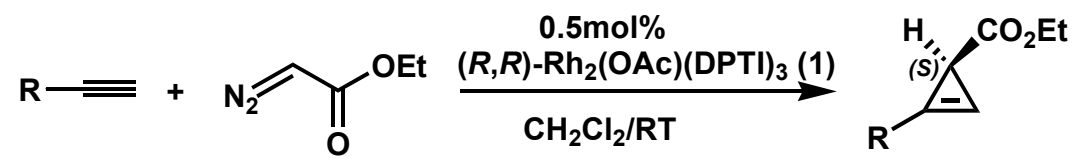

To a solution of alkyne $(1.9 \mathrm{mmol}, 10 \mathrm{eq})$ and $(R, R)-\mathrm{Rh}_{2}(\mathrm{OAc})(\mathrm{DPTI})_{3}(\mathbf{1})(0.00095$ mmol, 0.005 eq) in $\mathrm{CH}_{2} \mathrm{Cl}_{2}(1.8 \mathrm{~mL})$, was added ethyl diazoacetate $(0.19 \mathrm{mmol}, 1 \mathrm{eq})$ in $\mathrm{CH}_{2} \mathrm{Cl}_{2}(1.2 \mathrm{~mL})$ at room temperature through a syringe pump at a rate of $0.5 \mathrm{~mL} / \mathrm{h}$. After the addition was complete, the reaction mixture was filtered through a pipette column of neutral alumina, and flushed with $\mathrm{CH}_{2} \mathrm{Cl}_{2}$ to ensure complete elution of product. After removal of volatiles by rotary evaporation, the crude residue was purified by silica gel chromatography to provide the desired cyclopropene. Absolute configuration of the cyclopropene was determined by comparing the rotation sign with that previously reported. Enantiomeric excess was obtained either by gas chromatography or by HPLC on the specified chiral column.

(S)-Ethyl 2-pentylcycloprop-2-enecarboxylate (Table 1, entry 1) ${ }^{2}$

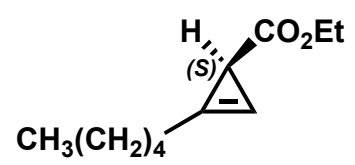

Purification by column chromatography (20:1 hexanes-EtOAc) afforded the above cyclopropene (31 mg, 90\%) as colorless liquid. The enantiomeric excess was determined to be $95 \%$ by $\mathrm{GC}\left(\gamma\right.$-TA column): $90{ }^{\circ} \mathrm{C} ; 25 \mathrm{psi} ; \mathrm{t}_{\mathrm{R}}=22.1 \mathrm{~min}$ (major), $\mathrm{t}_{\mathrm{R}}=23.9 \mathrm{~min}$ (minor); $[\alpha]^{23}{ }_{\mathrm{D}}=+76^{\circ}\left(c 0.6, \mathrm{CHCl}_{3}\right) ;{ }^{1} \mathrm{H} \mathrm{NMR}\left(\mathrm{CDCl}_{3}, 400 \mathrm{MHz}\right): \delta 6.32(\mathrm{~d}, J=1.6$ $\mathrm{Hz}, 1 \mathrm{H}), 4.12(\mathrm{~m}, 2 \mathrm{H}), 2.48(\mathrm{dt}, J=1.6,7.2 \mathrm{~Hz}, 2 \mathrm{H}), 2.12(\mathrm{~d}, J=1.6 \mathrm{~Hz}, 1 \mathrm{H}), 1.58$ (quintet, $J=6.8 \mathrm{~Hz}, 2 \mathrm{H}), 1.33(\mathrm{~m}, 4 \mathrm{H}), 1.25(\mathrm{t}, J=6.8 \mathrm{~Hz}, 3 \mathrm{H}), 0.89(\mathrm{t}, J=7.2 \mathrm{~Hz}, 3 \mathrm{H})$ 
ppm; ${ }^{13} \mathrm{C}$ NMR $\left(\mathrm{CDCl}_{3}, 400 \mathrm{MHz}\right): \delta 176.7,115.6,93.9,60.1,31.3,26.3,24.9,22.3$, 19.7, 14.4, 13.9 ppm; FTIR (film): 1712, 1180, 910, and $729 \mathrm{~cm}^{-1}$; HRMS $\left(\mathrm{CI}^{+}\right)$: calcd for $\mathrm{C}_{11} \mathrm{H}_{22} \mathrm{NO}_{2}\left(\mathrm{M}+\mathrm{NH}_{4}\right)^{+}$200.1651, found 200.1650.

(S)-Ethyl 2-hexylcycloprop-2-enecarboxylate (Table 1, entry 2)

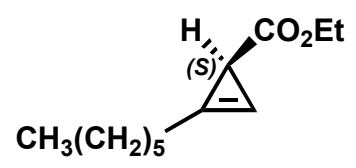

Purification by column chromatography (20:1 hexanes-EtOAc) afforded the above cyclopropene (34 mg, 90\%) as colorless liquid. The enantiomeric excess was determined to be $93 \%$ by GC (Cyclosil B column): $90{ }^{\circ} \mathrm{C} ; 25 \mathrm{psi} ; \mathrm{t}_{\mathrm{R}}=91.9 \mathrm{~min}$ (major), $\mathrm{t}_{\mathrm{R}}=89.9$ $\min ($ minor $) ;[\alpha]^{23}{ }_{\mathrm{D}}=+67^{\circ}\left(c 0.08, \mathrm{CHCl}_{3}\right) ;{ }^{1} \mathrm{H} \mathrm{NMR}\left(\mathrm{CDCl}_{3}, 400 \mathrm{MHz}\right): \delta 6.29(\mathrm{~d}, J=$ $1.2 \mathrm{~Hz}, 1 \mathrm{H}), 4.08(\mathrm{~m}, 2 \mathrm{H}), 2.45(\mathrm{dt}, J=1.2,7.6 \mathrm{~Hz}, 2 \mathrm{H}), 2.08(\mathrm{~d}, J=1.2 \mathrm{~Hz}, 1 \mathrm{H}), 1.54$ (quintet, $J=7.6 \mathrm{~Hz}, 2 \mathrm{H}), 1.26(\mathrm{~m}, 9 \mathrm{H}), 0.85 \mathrm{ppm}(\mathrm{t}, J=7.2 \mathrm{~Hz}, 3 \mathrm{H}) ;{ }^{13} \mathrm{C} \mathrm{NMR}\left(\mathrm{CDCl}_{3}\right.$, $400 \mathrm{MHz}): \delta 176.8,115.8,94.1,60.3,31.7,30.0,26.8,25.2,22.7,19.9,14.6,14.2 \mathrm{ppm}$; FTIR (film): 2929, 1717, 1177, 1030, $731 \mathrm{~cm}^{-1}$; $\left.\mathrm{HRMS} \mathrm{(ESI}{ }^{+}\right)$: calcd for $\mathrm{C}_{12} \mathrm{H}_{21} \mathrm{O}_{2}(\mathrm{M}+$ H) ${ }^{+}$197.1541, found 197.1543 .

(S)-Ethyl 2-(t-butyl)cycloprop-2-ene-1-carboxylate (Table 1, entry 3) ${ }^{2}$

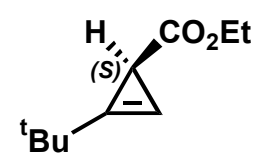

Purification by column chromatography (20:1 hexanes-EtOAc) afforded the above cyclopropene (26 mg, 81\%) as colorless liquid. The enantiomeric excess was determined to be $92 \%$ by GC (Cyclosil B column): $70{ }^{\circ} \mathrm{C} ; 25 \mathrm{psi} ; \mathrm{t}_{\mathrm{R}}=16.3 \mathrm{~min}$ (major), $\mathrm{t}_{\mathrm{R}}=17.7$ $\min ($ minor $) ;[\alpha]^{23}{ }_{\mathrm{D}}=+74^{\circ}\left(c 0.8, \mathrm{CHCl}_{3}\right) ;{ }^{1} \mathrm{H} \mathrm{NMR}\left(\mathrm{CDCl}_{3}, 400 \mathrm{MHz}\right): \delta 6.17(\mathrm{~d}, J=$ $1.6 \mathrm{~Hz}, 1 \mathrm{H}), 4.09$ (m, 2H), $2.12(\mathrm{~d}, J=1.6 \mathrm{~Hz}, 1 \mathrm{H}), 1.20(\mathrm{t}, J=7.6 \mathrm{~Hz}, 3 \mathrm{H}), 1.14$ (s, 9H) 
ppm; ${ }^{13} \mathrm{C}$ NMR $\left(\mathrm{CDCl}_{3}, 400 \mathrm{MHz}\right): \delta 176.5,123.2,91.0,59.9,31.1,27.7,19.7,14.3$ ppm.

(S)-Ethyl 2-(diethoxymethyl)cycloprop-2-ene-1-carboxylate (Table 1, entry 4) ${ }^{2}$

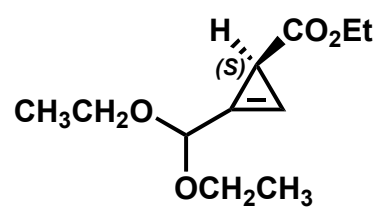

Purification by column chromatography (silica gel pretreated with $1 \% \mathrm{Et}_{3} \mathrm{~N}$ ) with 10:1 hexanes-EtOAc afforded the above cyclopropene (26 mg, 64\%) as colorless liquid. The enantiomeric excess was determined to be $92 \%$ by GC (Cyclosil B column): $100{ }^{\circ} \mathrm{C}$; $25 \mathrm{psi} ; \mathrm{t}_{\mathrm{R}}=30.7 \min ($ major $), \mathrm{t}_{\mathrm{R}}=29.4 \min ($ minor $) ;[\alpha]^{23}{ }_{\mathrm{D}}=+47^{\circ}\left(c 0.12, \mathrm{CHCl}_{3}\right) ;{ }^{1} \mathrm{H}$ NMR $\left(\mathrm{CDCl}_{3}, 400 \mathrm{MHz}\right): \delta 6.78(\mathrm{~d}, J=0.8 \mathrm{~Hz}, 1 \mathrm{H}), 5.49(\mathrm{~d}, J=0.8 \mathrm{~Hz}, 1 \mathrm{H}), 4.12(\mathrm{~m}$, 2H), $3.59(\mathrm{~m}, 4 \mathrm{H}), 2.34(\mathrm{~d}, J=0.8 \mathrm{~Hz}, 1 \mathrm{H}), 1.22(\mathrm{~m}, 9 \mathrm{H}) \mathrm{ppm} ;{ }^{13} \mathrm{C} \mathrm{NMR}\left(\mathrm{CDCl}_{3}, 400\right.$ MHz): $\delta .175 .3,112.9,100.7,95.9,61.8,61.5,21.1,15.4,14.5$ ppm; FTIR (film): 1721, 1186, $1053 \mathrm{~cm}^{-1}$; HRMS $\left(\mathrm{CI}^{+}\right)$: calcd for $\mathrm{C}_{11} \mathrm{H}_{22} \mathrm{NO}_{4}\left(\mathrm{M}+\mathrm{NH}_{4}\right)^{+}$232.1549, found 232.1550 .

(S)-Ethyl 2-(benzyloxymethyl)cycloprop-2-ene-1-carboxylate (Table 1, entry 5)

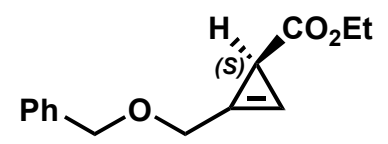

Purification by column chromatography (10:1 hexanes-EtOAc) afforded the above cyclopropene (38 mg, 86\%) as colorless liquid. The enantiomeric excess was determined to be $92 \%$ by GC (Cyclosil B column): $155^{\circ} \mathrm{C} ; 25 \mathrm{psi} ; \mathrm{t}_{\mathrm{R}}=30.4 \min$ (major), $\mathrm{t}_{\mathrm{R}}=30.1$ $\min \left(\right.$ minor); $[\alpha]^{23}{ }_{\mathrm{D}}=+11^{\circ}\left(c\right.$ 0.6, $\left.\mathrm{CHCl}_{3}\right) ;{ }^{1} \mathrm{H} \mathrm{NMR}\left(\mathrm{CDCl}_{3}, 400 \mathrm{MHz}\right): \delta 7.35(\mathrm{~m}, 5 \mathrm{H})$, $6.67(\mathrm{~d}, J=1.2 \mathrm{~Hz}, 1 \mathrm{H}), 4.61(\mathrm{~d}, J=1.2 \mathrm{~Hz}, 2 \mathrm{H}), 4.54(\mathrm{~s}, 2 \mathrm{H}), 4.14(\mathrm{~m}, 2 \mathrm{H}), 2.31(\mathrm{~d}, J=$ $1.2 \mathrm{~Hz}, 1 \mathrm{H}), 1.25(\mathrm{t}, J=7.0 \mathrm{~Hz}, 3 \mathrm{H}) \mathrm{ppm} ;{ }^{13} \mathrm{C} \mathrm{NMR}\left(\mathrm{CDCl}_{3}, 400 \mathrm{MHz}\right): \delta 175.8,137.7$, 
128.7, 128.2, 128.1, 112.6, 97.9, 72.8, 63.8, 60.7, 20.4, 14.6 ppm; FTIR (film): 1712, 1182, 1091, 1026, 730, $697 \mathrm{~cm}^{-1}$; HRMS $\left(\mathrm{ESI}^{+}\right)$: calcd for $\mathrm{C}_{14} \mathrm{H}_{17} \mathrm{O}_{3}(\mathrm{M}+\mathrm{H})^{+} 233.1177$, found 233.1174 .

(S)-Ethyl 2-(methyoxymethyl)cycloprop-2-ene-1-carboxylate (Table 1, entry 6) ${ }^{2}$

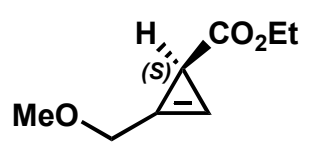

Purification by column chromatography (20:1 hexanes-EtOAc) afforded the above cyclopropene ( $23 \mathrm{mg}, 78 \%)$ as colorless liquid. The enantiomeric excess was determined to be $92 \%$ by GC $\left(\gamma\right.$-TA column): $80^{\circ} \mathrm{C} ; 25 \mathrm{psi} ; \mathrm{t}_{\mathrm{R}}=28.2 \min$ (major), $\mathrm{t}_{\mathrm{R}}=29.5 \mathrm{~min}$ (minor); $[\alpha]^{23}{ }_{\mathrm{D}}=+57^{\circ}\left(c \mathrm{0.3}, \mathrm{CHCl}_{3}\right) ;{ }^{1} \mathrm{H} \mathrm{NMR}\left(\mathrm{CDCl}_{3}, 400 \mathrm{MHz}\right): \delta 6.64(\mathrm{dd}, J=1.6$, $3.2 \mathrm{~Hz}, 1 \mathrm{H}), 4.42(\mathrm{~d}, J=1.6 \mathrm{~Hz}, 2 \mathrm{H}), 4.11(\mathrm{~m}, 2 \mathrm{H}), 3.39(\mathrm{~s}, 3 \mathrm{H}), 2.26(\mathrm{~d}, J=1.6 \mathrm{~Hz}, 1 \mathrm{H})$, $1.22(\mathrm{t}, J=7.20 \mathrm{~Hz}, 3 \mathrm{H}) \mathrm{ppm} ;{ }^{13} \mathrm{C} \mathrm{NMR}\left(\mathrm{CDCl}_{3}, 400 \mathrm{MHz}\right): \delta 175.5,112.2,97.4,65.90$, $60.3,58.5,19.9,14.2 \mathrm{ppm}$.

(S)-Ethyl 2-bromomethyl-cycloprop-2-enecarboxylate (Table 1, entry 7) ${ }^{3}$

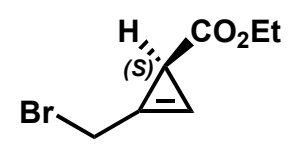

Purification by column chromatography (20:1 hexanes-EtOAc) afforded the above cyclopropene (24 mg, 62\%) as colorless liquid. The enantiomeric excess was determined to be $95 \%$ by $\mathrm{GC}\left(\gamma-\mathrm{TA}\right.$ column): $80^{\circ} \mathrm{C}, 25 \mathrm{psi} ; \mathrm{t}_{\mathrm{R}}=67.6 \mathrm{~min}$ (major), $\mathrm{t}_{\mathrm{R}}=64.8 \mathrm{~min}$ (minor); $[\alpha]^{23}{ }_{\mathrm{D}}=+70^{\circ}\left(c \mathrm{1} 15, \mathrm{CHCl}_{3}\right) ;{ }^{1} \mathrm{H} \mathrm{NMR}\left(\mathrm{CDCl}_{3}, 400 \mathrm{MHz}\right): \delta 6.66(\mathrm{~d}, J=1.2$ $\mathrm{Hz}, 1 \mathrm{H}), 4.30(\mathrm{dd}, J=1.2,12.8 \mathrm{~Hz}, 1 \mathrm{H}), 4.22(\mathrm{dd}, J=1.2,12.8 \mathrm{~Hz}, 1 \mathrm{H}), 4.11(\mathrm{~m}, 2 \mathrm{H})$, $2.35(\mathrm{~d}, J=1.2 \mathrm{~Hz}), 1.22(\mathrm{t}, J=8.0 \mathrm{~Hz}, 3 \mathrm{H}) \mathrm{ppm} ;{ }^{13} \mathrm{C} \mathrm{NMR}\left(\mathrm{CDCl}_{3}, 400 \mathrm{MHz}\right): \delta 174.6$, $111.7,99.6,60.5,22.4,21.0,14.2 \mathrm{ppm}$. 
(S)-Ethyl 2-(3-phenyl-allyloxymethyl)-cycloprop-2-ene-1-carboxylate (Table 1, entry

8)

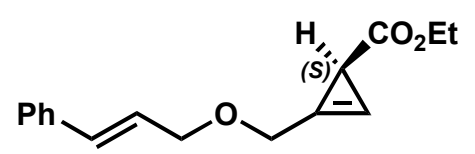

This reaction was performed in pentane as solvent because the yield was only around $20 \%$ when the reaction was done in dichloromethane. Purification by column chromatography (20:1 hexanes-EtOAc) afforded the above cyclopropene (24 $\mathrm{mg}, 76 \%)$ as colorless liquid. The enantiomeric excess was determined to be $95 \%$ by HPLC (Chiralcel OJ column): $5 \%$ isopropanol in hexanes as eluent; flow rate $=0.9 \mathrm{~mL} / \mathrm{min} ; \lambda=$ $254 \mathrm{~nm} ; \mathrm{t}_{\mathrm{R}}=71.9 \min ($ major $), \mathrm{t}_{\mathrm{R}}=86.0 \min (\operatorname{minor}) ;[\alpha]^{23}{ }_{\mathrm{D}}=+25^{\circ}\left(c 1.65, \mathrm{CHCl}_{3}\right) ;{ }^{1} \mathrm{H}$ $\operatorname{NMR}\left(\mathrm{CDCl}_{3}, 400 \mathrm{MHz}\right): \delta 7.39(\mathrm{~d}, J=7.2 \mathrm{~Hz}, 2 \mathrm{H}), 7.32(\mathrm{t}, J=7.2 \mathrm{~Hz}, 2 \mathrm{H}), 7.25(\mathrm{~d}, J=$ $7.2 \mathrm{~Hz}, 1 \mathrm{H}), 6.68(\mathrm{~d}, J=1.6 \mathrm{~Hz}, 1 \mathrm{H}), 6.63(\mathrm{~d}, J=16 \mathrm{~Hz}, 1 \mathrm{H}), 6.29$ (td, $J=6.4,16 \mathrm{~Hz})$, $4.56(\mathrm{~d}, J=1.6 \mathrm{~Hz}, 2 \mathrm{H}), 4.25(\mathrm{td}, J=1.2,6.4 \mathrm{~Hz}, 2 \mathrm{H}), 4.14(\mathrm{~m}, 2 \mathrm{H}), 2.32(\mathrm{~d}, J=1.6 \mathrm{~Hz}$, $1 \mathrm{H}), 1.25(\mathrm{t}, J=6.8 \mathrm{~Hz}, 3 \mathrm{H}) \mathrm{ppm} ;{ }^{13} \mathrm{C} \mathrm{NMR}\left(\mathrm{CDCl}_{3}, 400 \mathrm{MHz}\right): \delta 175.6,136.4,133.2$, 128.6, 127.8, 126.5, 125.1, 112.4, 97.6, 71.1, 63.4, 60.4, 20.2, 14.3 ppm; FTIR (film): 1716, 1187, 736, $694 \mathrm{~cm}^{-1}$; HRMS $\left(\mathrm{CI}^{+}\right)$: calcd for $\mathrm{C}_{16} \mathrm{H}_{22} \mathrm{NO}_{3}$ 276.1600, found 276.1605 .

\section{Part 3. Cyclopropanation and Other Reactions Catalyzed by 1}

Cyclopropanation of styrene with ethyl diazoacetate

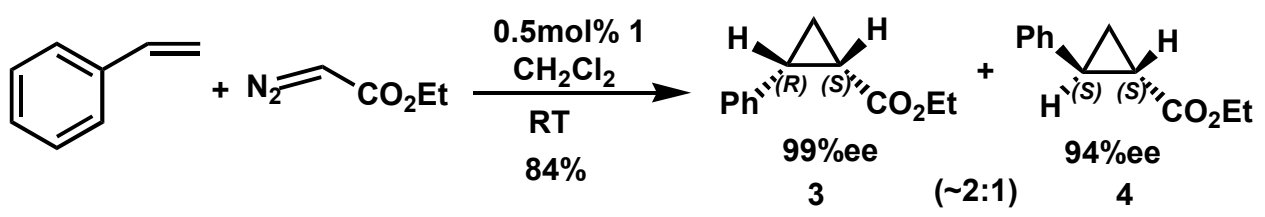


The procedure is identical to the cyclopropenation described in Part 2. The crude mixture was purified with preparative thin-layer chromatography (10:1 hexanes-EtOAc) to give $9.5 \mathrm{mg}(56 \%)$ of the cis-cyclopropane (3) and $4.5 \mathrm{mg}(28 \%)$ of the transcyclopropane (4). ${ }^{4,5}$ Diagnostic data for the cis-cyclopropane 3: ${ }^{1} \mathrm{H}$ NMR $\left(\mathrm{CDCl}_{3}, 400\right.$ MHz): $\delta 7.27(\mathrm{~m}, 4 \mathrm{H}), 7.20(\mathrm{~m}, 1 \mathrm{H}), 3.87(\mathrm{q}, J=6.8 \mathrm{~Hz}, 2 \mathrm{H}), 2.58(\mathrm{q}, J=8.0 \mathrm{~Hz}, 2 \mathrm{H})$, $2.08(\mathrm{~m}, 1 \mathrm{H}), 1.72(\mathrm{~m}, 1 \mathrm{H}), 1.33(\mathrm{~m}, 1 \mathrm{H}), 0.97(\mathrm{t}, J=6.8 \mathrm{~Hz}, 3 \mathrm{H}) \mathrm{ppm} ;[\alpha]^{23}{ }_{\mathrm{D}}=+48^{\circ}(c$ 1.84, $\left.\mathrm{CHCl}_{3}\right)$. Diagnostic data for the trans-cyclopropane $4:{ }^{1} \mathrm{H} \mathrm{NMR}\left(\mathrm{CDCl}_{3}, 400 \mathrm{MHz}\right)$ : $\delta 7.28(\mathrm{t}, J=7.6 \mathrm{~Hz}, 2 \mathrm{H}), 7.21(\mathrm{t}, J=7.6 \mathrm{~Hz}, 1 \mathrm{H}), 7.10(\mathrm{~d}, J=7.6 \mathrm{~Hz}, 2 \mathrm{H}), 4.17(\mathrm{q}, J=$ $7.2 \mathrm{~Hz}, 2 \mathrm{H}), 2.52(\mathrm{~m}, 1 \mathrm{H}), 1.90(\mathrm{~m}, 1 \mathrm{H}), 1.60(\mathrm{~m}, 1 \mathrm{H}), 1.31(\mathrm{~m}, 1 \mathrm{H}), 1.28(\mathrm{t}, J=7.2 \mathrm{~Hz}$, 3H) ppm; $[\alpha]_{D}^{23}=+14^{\circ}\left(c 2.33, \mathrm{CHCl}_{3}\right)$. The absolute configuration of compounds 3 and 4 was determined by comparison of the sign of their optical rotations with that reported. ${ }^{4}$ The enantiomeric excess was determined to be $99 \%$ for the cis-cyclopropane and $94 \%$ for the trans-cyclopropane by GC (Cyclosil B column): $130{ }^{\circ} \mathrm{C} ; 25 \mathrm{psi} ; \mathrm{t}_{\mathrm{R}}=30.3 \mathrm{~min}$ (major isomer of 3$), t_{R}=32.5 \min ($ minor isomer of 3$), t_{R}=40.8$ min (major isomer of 4$), t_{R}=$ $40.1 \mathrm{~min}$ (minor isomer of 4 ).

\section{Cyclopropanation of ethyl diazoacetate and 1,1-diphenylethylene}

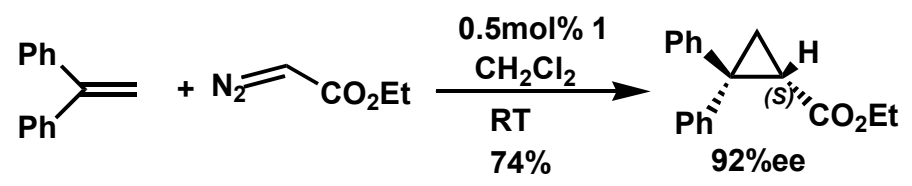

5

The procedure is identical to the cyclopropenation described in Part 2. The crude mixture was purified by preparative thin-layer chromatography $(10: 1$ hexanes-EtOAc) to give cyclopropane $5^{5}(52 \mathrm{mg}, 74 \%)$ as colorless liquid. ${ }^{1} \mathrm{H} \mathrm{NMR}\left(\mathrm{CDCl}_{3}, 500 \mathrm{MHz}\right): \delta$ $7.82(\mathrm{~d}, J=7.5 \mathrm{~Hz}, 2 \mathrm{H}), 7.60(\mathrm{t}, J=7.5 \mathrm{~Hz}, 1 \mathrm{H}), 7.49(\mathrm{t}, J=7.5 \mathrm{~Hz}, 2 \mathrm{H}), 7.35(\mathrm{~d}, J=7.5$ 
$\mathrm{Hz}, 2 \mathrm{H}), 7.3-7.1(\mathrm{~m}, 3 \mathrm{H}), 3.91(\mathrm{~m}, 2 \mathrm{H}), 2.54(\mathrm{dd}, J=6.0,8.5 \mathrm{~Hz}, 1 \mathrm{H}), 2.18(\mathrm{dd}, J=5.0$, $6.0 \mathrm{~Hz}, 1 \mathrm{H}), 1.59(\mathrm{dd}, J=5.0,8.5 \mathrm{~Hz}, 1 \mathrm{H}), 1.01(\mathrm{t}, J=7.0 \mathrm{~Hz}, 3 \mathrm{H}) \mathrm{ppm}$; The enantiomeric excess was determined to be $92 \%$ by HPLC $((S, S)$ Whelk-O1 column): 1\% isopropanol in hexanes as eluent; flow rate $=1.0 \mathrm{~mL} / \mathrm{min} ; \mathrm{t}_{\mathrm{R}}=15.6 \mathrm{~min}$ (major), $\mathrm{t}_{\mathrm{R}}=$ 14.9 min (minor); The ester 5 was reduced to $1 S$-(2,2-diphenyl-cylopropyl)-methanol 4 $\left([\alpha]^{23}{ }_{\mathrm{D}}=+181^{\circ}\left(\mathrm{c} 0.33, \mathrm{CH}_{2} \mathrm{Cl}_{2}\right)\right)$ with $\mathrm{LAH}$ in $\mathrm{Et}_{2} \mathrm{O}$ at room temperature in order to determine the absolute configuration.

\section{Competition experiments with a mixture of 4-methoxystyrene and 4-nitrostyrene}
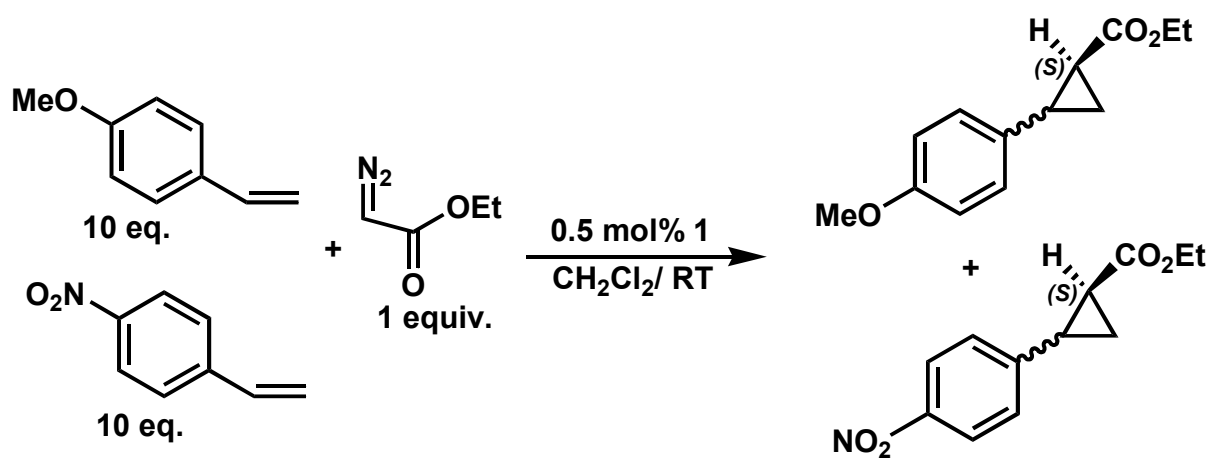

To a solution of 4-methoxystyrene ( $0.78 \mathrm{mmol}, 10 \mathrm{eq})$, 4-nitrostyrene $(0.78 \mathrm{mmol}, 10$ eq) and $(R, R)-\mathrm{Rh}_{2}(\mathrm{OAc})(\mathrm{DPTI})_{3}(\mathbf{1})\left(0.00039 \mathrm{mmol}, 0.005\right.$ eq) in $\mathrm{CH}_{2} \mathrm{Cl}_{2}(1 \mathrm{~mL})$, ethyl diazoacetate $(0.078 \mathrm{mmol}, 1 \mathrm{eq})$ in $\mathrm{CH}_{2} \mathrm{Cl}_{2}(1 \mathrm{~mL})$ was added at room temperature through a syringe pump at the rate of $0.1 \mathrm{~mL} / \mathrm{h}$. After the addition was complete, the reaction mixture was filtered through a pipette column of neutral alumina, which was flushed with $\mathrm{CH}_{2} \mathrm{Cl}_{2}$ to ensure complete transfer of product. This crude mixture was analyzed by $\mathrm{GC}$ (Cyclosil B column): $110^{\circ} \mathrm{C}$ for 8 min then $2{ }^{\circ} \mathrm{C} / \mathrm{min}$ to $180{ }^{\circ} \mathrm{C}$ then 180 ${ }^{\circ} \mathrm{C}$ for $30 \mathrm{~min} ; 25 \mathrm{psi} ; \mathrm{t}_{\mathrm{R}}=35.36 \mathrm{~min}$ (major cis-cyclopropane from 4-methoxystyrene), $\mathrm{t}_{\mathrm{R}}$ $=35.74 \mathrm{~min}$ (minor cis-cyclopropane from 4-methoxystyrene), $\mathrm{t}_{\mathrm{R}}=39.10 \mathrm{~min}$ (transcyclopropanes from 4-methoxystyrene), $t_{R}=51.77$ min (major cis-cyclopropane from 4- 
nitrostyrene), $t_{R}=52.11$ min (minor cis-cyclopropane from 4-nitrostyrene), $t_{R}=58.86$ min (trans-cyclopropanes from 4-nitrostyrene). The ratio of cyclopropanes from 4methoxystyrene versus cyclopropanes from 4-nitrostyrene was determined to be $\sim 1.7: 1$. The cis/trans ratio of diastereoisomeric cyclopropanes from 4-methoxystyrene and 4nitrostyrene was found to be 2.3 and 2.2 respectively. The enantiomeric excess for both of the cis-cyclopropyl esters was $97 \%$. The ratios of cyclopropanes were determined solely on the relative GC areas without correction of relative response factors.

(2S,3R)-2,3-cis-3-Methoxycarbonyl-2-phenyl-2,3-dihydrobenzofuran (7) ${ }^{6}$

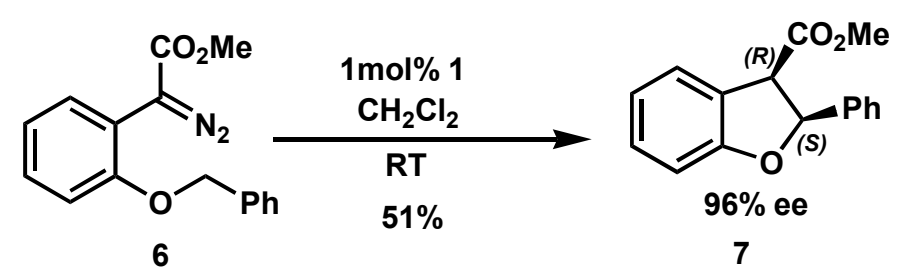

To a solution of $(R, R)-\mathrm{Rh}_{2}(\mathrm{OAc})(\mathrm{DPTI})_{3}(\mathbf{1})(1.5 \mathrm{mg}, 0.0011 \mathrm{mmol})$ in $\mathrm{CH}_{2} \mathrm{Cl}_{2}(1$ $\mathrm{mL})$, a solution of diazo ester $6{ }^{6}(30 \mathrm{mg}, 0.11 \mathrm{mmol})$ in $\mathrm{CH}_{2} \mathrm{Cl}_{2}(0.5 \mathrm{~mL})$ was added at room temperature through a syringe pump at the rate of $0.1 \mathrm{~mL} / \mathrm{h}$. After addition, the reaction mixture was allowed to stir at room temperature for another $6 \mathrm{~h}$ before being concentrated in vacuo. The cis/trans ratio of 7 was determined to be $5: 1$ by ${ }^{1} \mathrm{H}$ NMR of the crude product. Purification by column chromatography (10:1 hexanes-EtOAc) gave cis-7 (14 mg, 51\%) as a colorless solid. The ${ }^{1} \mathrm{H}$ NMR is in accordance with that reported in literature. ${ }^{6}$ The absolute configuration was determined to be opposite to that reported ${ }^{6}$ by comparison of the order of elution of the major isomer. The enantiomeric excess of 7 was determined to be $96 \%$ by HPLC (Daicel Chiralcel OD-H column): $10 \%$ isopropanol in hexanes as eluent; flow rate $=1.0 \mathrm{~mL} / \mathrm{min} ; \mathrm{t}_{\mathrm{R}}=19.0 \mathrm{~min}$ (major), $\mathrm{t}_{\mathrm{R}}=9.5 \mathrm{~min}$ (minor). 


\section{Part 4. Transformations of Ethyl (1S)-2-n-Pentyl-2-cyclopropene-carboxylate} Cycloaddition reaction with cyclopentadiene $^{7}$

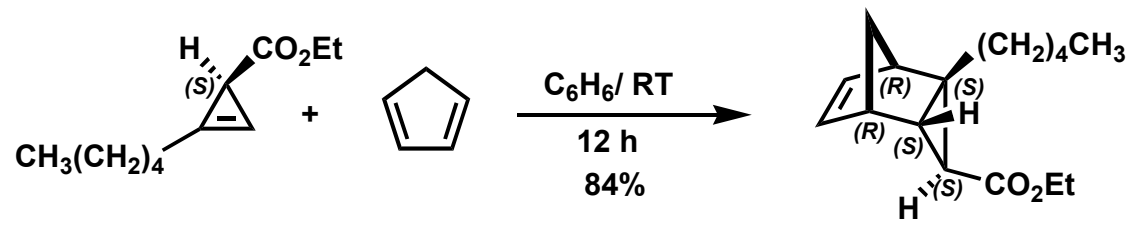

A solution of ethyl (1S)-2-n-pentyl-2-cyclopropene-carboxylate (40 mg, $0.22 \mathrm{mmol})$ and cyclopentadiene $(145 \mathrm{mg}, 2.20 \mathrm{mmol})$ in benzene $(2 \mathrm{~mL})$ was stirred at room temperature for $12 \mathrm{~h}$. The residue after rotary evaporation was purified by column chromatography (20:1 hexanes-EtOAc) to afford the desired cycloadduct (51 mg, 94\%) as colorless liquid: $[\alpha]^{23}{ }_{\mathrm{D}}=+66^{\circ}\left(c 0.35, \mathrm{CHCl}_{3}\right) ;{ }^{1} \mathrm{H}$ NMR $\left(\mathrm{CDCl}_{3}, 500 \mathrm{MHz}\right): \delta 5.92$ $(\mathrm{dd}, J=3.5,4.5 \mathrm{~Hz}, 1 \mathrm{H}), 5.84(\mathrm{dd}, J=3.5,4.5 \mathrm{~Hz}, 1 \mathrm{H}), 4.07(\mathrm{~m}, 2 \mathrm{H}), 2.91(\mathrm{~m}, 1 \mathrm{H}), 2.77$ $(\mathrm{m}, 1 \mathrm{H}), 1.91(\mathrm{~m}, 1 \mathrm{H}), 1.87(\mathrm{~m}, 2 \mathrm{H}), 1.65(\mathrm{~m}, 1 \mathrm{H}), 1.59(\mathrm{~d}, J=7.0 \mathrm{~Hz}, 1 \mathrm{H}), 1.48(\mathrm{~d}, J=$ $3.5 \mathrm{~Hz}, 1 \mathrm{H}), 1.43(\mathrm{~m}, 1 \mathrm{H}), 1.32-1.14(\mathrm{~m}, 8 \mathrm{H}), 0.87(\mathrm{t}, J=7.0 \mathrm{~Hz}, 3 \mathrm{H}) \mathrm{ppm} ;{ }^{13} \mathrm{C} \mathrm{NMR}$ $\left(\mathrm{CDCl}_{3}, 500 \mathrm{MHz}\right): \delta 171.4,132.8,132.8,62.0,60.3,48.9,43.6,35.2,34.2,32.2,29.2$ 28.7, 26.7, 22.9, 14.5, and 14.3 ppm; FTIR (film): 1720, 1454, 1401, 1315, 1193, 1168 $1046 \mathrm{~cm}^{-1}$; HRMS $\left(\mathrm{ES}^{+}\right)$calcd for $\mathrm{C}_{16} \mathrm{H}_{25} \mathrm{O}_{2}(\mathrm{M}+\mathrm{H})^{+}$249.1854, found 249.1852.

\section{Cycloaddition reaction with 2,3-dimethylbutadiene}

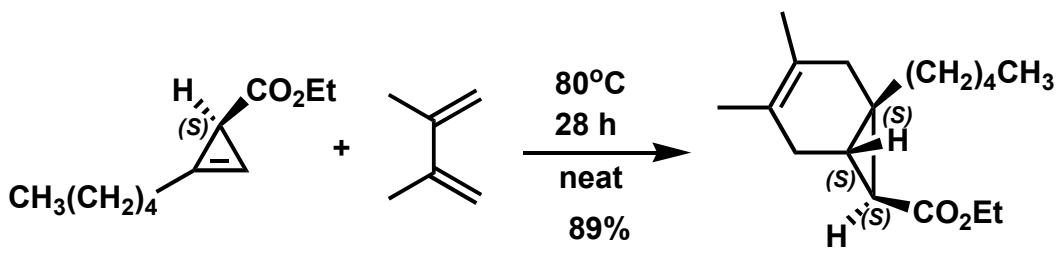

A solution of ethyl (1S)-2-n-pentyl-2-cyclopropene-carboxylate (17 mg, $0.093 \mathrm{mmol})$ in 2,3-dimethylbutadiene $(0.2 \mathrm{~mL})$ was heated at $80{ }^{\circ} \mathrm{C}$ in a sealed tube for $28 \mathrm{~h}$. After being cooled to room temperature, the mixture was concentrated and purified with 
column chromatography (30:1 hexanes-EtOAc) to provide the desired adduct $(22 \mathrm{mg}$, 89\%) as colorless liquid: $[\alpha]^{23}{ }_{\mathrm{D}}=+52^{\circ}\left(c 1.1, \mathrm{CHCl}_{3}\right) ;{ }^{1} \mathrm{H} \mathrm{NMR}\left(\mathrm{CDCl}_{3}, 500 \mathrm{MHz}\right): \delta$ $4.10(\mathrm{q}, J=7.5 \mathrm{~Hz}, 2 \mathrm{H}), 2.36(\mathrm{~d}, J=17.5 \mathrm{~Hz}, 1 \mathrm{H}), 2.26(\mathrm{~d}, J=17.5 \mathrm{~Hz}, 1 \mathrm{H}), 2.16(\mathrm{~d}, J=$ $17.5 \mathrm{~Hz}, 2 \mathrm{H}), 1.7-1.5(\mathrm{~m}, 10 \mathrm{H}), 1.41(\mathrm{~m}, 1 \mathrm{H}), 1.3-1.1(\mathrm{~m}, 8 \mathrm{H}), 0.86(\mathrm{t}, J=7.5 \mathrm{~Hz}, 3 \mathrm{H})$ ppm; ${ }^{13} \mathrm{C}$ NMR $\left(\mathrm{CDCl}_{3}, 500 \mathrm{MHz}\right): \delta$ 173.8, 122.1, 121.8, 60.3, 36.0, 33.1, 32.3, 32.1, 30.9, 28.0, 27.0, 26.6, 22.9, 19.4, 19.2, 14.6, 14.3 ppm; FTIR (film): 2857, 1720, 1451, $1369,1348,1295,1244,1224,1153 \mathrm{~cm}^{-1}$; HRMS $\left(\mathrm{ESI}^{+}\right)$calcd for $\mathrm{C}_{17} \mathrm{H}_{28} \mathrm{O}_{2}(\mathrm{M}+\mathrm{H})^{+}$ 265.2163 , found 265.2163 .

((1S,2S)-2-Pentylcyclopropyl)methanol ${ }^{8}$

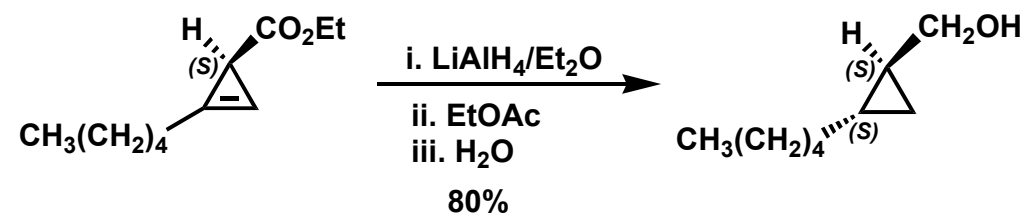

Lithium aluminum hydride ( $13 \mathrm{mg}, 0.34 \mathrm{mmol})$ was added in one portion at $0{ }^{\circ} \mathrm{C}$ to a solution of ethyl (1S)-2-n-pentyl-2-cyclopropene-carboxylate (40 mg, $0.22 \mathrm{mmol})$ in ether $(2 \mathrm{~mL})$. The suspension was warmed up to room temperature and stirred for $2 \mathrm{~h}$ before the sequential treatment with ethyl acetate and water. The resulting mixture was extracted with ether $(20 \mathrm{~mL} \times 2)$, washed with brine $(20 \mathrm{~mL})$, and dried with $\mathrm{MgSO}_{4}$. After removal of volatiles by rotary evaporation, the residue was purified by column chromatography (6:1 hexanes-EtOAc) to give the desired alcohol $(25 \mathrm{mg}, 80 \%)$ as colorless liquid: $[\alpha]^{23}=+40^{\circ}\left(c 0.20, \mathrm{CHCl}_{3}\right) ;{ }^{1} \mathrm{H} \mathrm{NMR}\left(\mathrm{CDCl}_{3}, 400 \mathrm{MHz}\right): \delta 3.43(\mathrm{~m}$, 2H), $1.29(\mathrm{~m}, 8 \mathrm{H}), 0.88(\mathrm{t}, J=7.2 \mathrm{~Hz}, 3 \mathrm{H}), 0.83(\mathrm{~m}, 1 \mathrm{H}), 0.59(\mathrm{~m}, 1 \mathrm{H}), 0.36(\mathrm{dt}, J=4.8$, $8.4 \mathrm{~Hz}, 1 \mathrm{H}), 0.30(\mathrm{dt}, J=4.8,8.4 \mathrm{~Hz}, 1 \mathrm{H}) \mathrm{ppm} ;{ }^{13} \mathrm{C} \mathrm{NMR}\left(\mathrm{CDCl}_{3}, 400 \mathrm{MHz}\right): \delta 67.3$, 
33.5, 31.6, 29.3, 22.6, 21.2, 17.2, 14.1, 9.90 ppm; FTIR (film): 2924, 1163, $1029 \mathrm{~cm}^{-1}$; HRMS $\left(\mathrm{CI}^{+}\right)$calcd for $\mathrm{C}_{9} \mathrm{H}_{22} \mathrm{NO}\left(\mathrm{M}+\mathrm{NH}_{4}\right)^{+}$160.1701, found 160.1699 .

\section{((1S,2S,3R)-2-Iodo-3-pentylcyclopropyl)methanol}

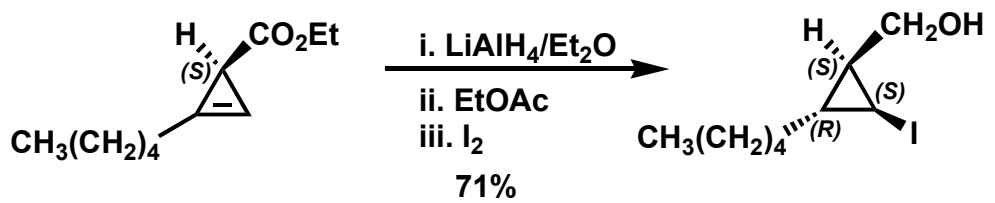

Lithium aluminum hydride $(62 \mathrm{mg}, 1.63 \mathrm{mmol})$ was added in one portion at $0{ }^{\circ} \mathrm{C}$ to a solution of ethyl (1S)-2-n-pentyl-2-cyclopropene-carboxylate (148 $\mathrm{mg}, 0.813 \mathrm{mmol})$ in ether $(2 \mathrm{~mL})$. The suspension was warmed up to room temperature and stirred for $2 \mathrm{~h}$. The reaction mixture was treated first with ethyl acetate $\left(0.1 \mathrm{~mL}\right.$, freshly distilled from $\left.\mathrm{CaH}_{2}\right)$ then cooled to $0{ }^{\circ} \mathrm{C}$. A solution of iodine $(0.83 \mathrm{~g}, 3.3 \mathrm{mmol})$ in ether $(2 \mathrm{~mL})$ was added dropwise via syringe. The resulting mixture was warmed up to room temperature and stirred for another hour. The brown reaction mixture was diluted with ether $(20 \mathrm{~mL})$, and washed with saturated $\mathrm{Na}_{2} \mathrm{~S}_{2} \mathrm{O}_{3}$ solution $(20 \mathrm{~mL} \times 2)$ and brine $(20 \mathrm{~mL})$. The organic layer was dried with anhydrous $\mathrm{MgSO}_{4}$. After removal of volatiles by rotary evaporation, the residue was purified by column chromatography (6:1 hexanes-EtOAc) to give the desired iodide $(155 \mathrm{mg}, 71 \%)$ as pale yellow liquid: $[\alpha]^{23}{ }_{\mathrm{D}}=-5.8^{\circ}\left(c\right.$ 2.8, $\left.\mathrm{CHCl}_{3}\right) ;{ }^{1} \mathrm{H}$ NMR ( $\left.\mathrm{CDCl}_{3}, 500 \mathrm{MHz}\right): \delta 3.91(\mathrm{~m}, 1 \mathrm{H}), 3.49(\mathrm{~m}, 1 \mathrm{H}), 2.38(\mathrm{dd}, J=4.5,7.5 \mathrm{~Hz}, 1 \mathrm{H})$, $1.75(\mathrm{dd}, J=4.5,7.5 \mathrm{~Hz}, 1 \mathrm{H}), 1.42-1.22(\mathrm{~m}, 8 \mathrm{H}), 0.98$ (quintet, $J=6.5 \mathrm{~Hz}, 1 \mathrm{H}), 0.89$ (t, $J$ $=6.5 \mathrm{~Hz}, 1 \mathrm{H}$ ), 0.70 (quintet, $J=4.5 \mathrm{~Hz}, 1 \mathrm{H}) \mathrm{ppm} ;{ }^{13} \mathrm{C} \mathrm{NMR}\left(\mathrm{CDCl}_{3}, 400 \mathrm{MHz}\right): \delta 68.0$, 32.2, 31.7, 28.5, 28.1, 24.9, 22.8, 14.3, -4.01 ppm; FTIR (film): 3339 (br), 2919, 2852, $1461,1453,1414,1378,1233,11.81,1037 \mathrm{~cm}^{-1}$; HRMS $\left(\mathrm{CI}^{+}\right)$calcd for $\mathrm{C}_{9} \mathrm{H}_{21} \mathrm{NIO}(\mathrm{M}+$ $\left.\mathrm{NH}_{4}\right)^{+} 286.0668$, found 286.0667 .

\section{((S)-2-Pentylcycloallyl)methanol ${ }^{9}$}




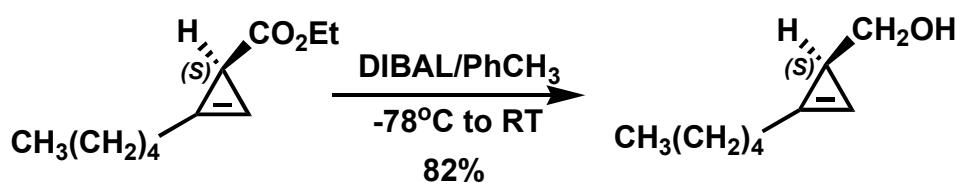

Di-isobutyl aluminum hydride ( $1 \mathrm{M}$ in toluene, $0.266 \mathrm{~mL}$ ) was added at $-78^{\circ} \mathrm{C}$ to the solution of ethyl (1S)-2-n-pentyl-2-cyclopropene-carboxylate $(23 \mathrm{mg}, 0.13 \mathrm{mmol})$ in toluene $(1 \mathrm{~mL})$. The resulting solution was stirred at $-78{ }^{\circ} \mathrm{C}$ for $1 \mathrm{~h}$ then at room temperature for $1 \mathrm{~h}$. The reaction mixture was treated with $1 \mathrm{~N} \mathrm{HCl}$ solution $(20 \mathrm{~mL})$ and extracted with ether $(20 \mathrm{ml} \times 2)$. The ether extraction was washed with brine $(20 \mathrm{~mL})$, dried with anhydrous $\mathrm{MgSO}_{4}$ and concentrated in vacuo. The residue was purified by column chromatography (6:1 hexanes-EtOAc) to give the desired product (15 $\mathrm{mg}, 83 \%)$ as a colorless liquid: $[\alpha]^{23}{ }_{\mathrm{D}}=+40^{\circ}\left(c 0.20, \mathrm{CHCl}_{3}\right) ;{ }^{1} \mathrm{H} \mathrm{NMR}\left(\mathrm{CDCl}_{3}, 400 \mathrm{MHz}\right): \delta 6.63$ (d, $J=0.8 \mathrm{~Hz}, 1 \mathrm{H}), 3.58(\mathrm{dd}, J=4.0,10.4 \mathrm{~Hz}, 1 \mathrm{H}), 3.46(\mathrm{dd}, J=4.0,10.4 \mathrm{~Hz}, 1 \mathrm{H}), 2.48$ (t, $J=7.2 \mathrm{~Hz}, 1 \mathrm{H}), 1.69(\mathrm{dd}, J=1.6,7.2 \mathrm{~Hz}, 1 \mathrm{H}), 1.58(\mathrm{~m}, 2 \mathrm{H}), 1.34(\mathrm{~m}, 4 \mathrm{H}), 0.90(\mathrm{t}, J=$ $7.2 \mathrm{~Hz}, 3 \mathrm{H}) \mathrm{ppm} ;{ }^{13} \mathrm{C} \mathrm{NMR}\left(\mathrm{CDCl}_{3}, 400 \mathrm{MHz}\right): \delta$ 125.8, 101.6, 68.7, 31.4, 26.9, 26.3, 22.4, 20.4, 14.0 ppm; FTIR (film): 2922, 2856, 1457, 1379, 1164, $1013 \mathrm{~cm}^{-1}$; HRMS $\left(\mathrm{Cl}^{+}\right)$calcd for $\mathrm{C}_{9} \mathrm{H}_{20} \mathrm{NO}\left(\mathrm{M}+\mathrm{NH}_{4}\right)^{+} 158.1545$, found 158.1548 .

\section{$(1 S, 2 R)$-Ethyl 2-pentylcyclopropanecarboxylate ${ }^{10}$}

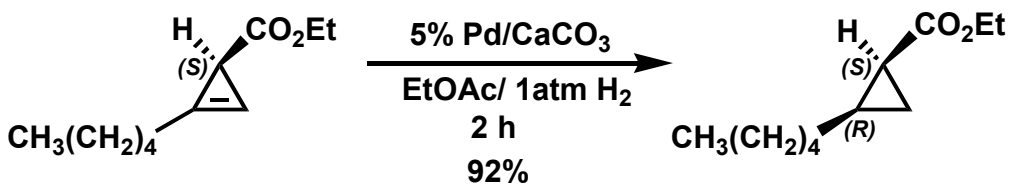

5\% $\mathrm{Pd}$ on $\mathrm{CaCO}_{3}(14 \mathrm{mg}, 0.0058 \mathrm{mmol})$ was added to a solution of ethyl (1S)-2-npentyl-2-cyclopropene-carboxylate $(105 \mathrm{mg}, 0.577 \mathrm{mmol})$ in ethyl acetate $(1.5 \mathrm{~mL})$ after being purged with hydrogen. The suspension was stirred vigorously under hydrogen atmosphere for $2 \mathrm{~h}$ when TLC indicated the complete consumption of the starting 
material. The reaction mixture was then filtered through a short plug of celite, and concentrated in vacuo. The residue was purified with column chromatography (40:1 hexanes-EtOAc) to give the desired cyclopropane $(97 \mathrm{mg}, 92 \%)$ as colorless liquid: $[\alpha]^{23}{ }_{\mathrm{D}}=+32^{\circ}\left(c 0.40, \mathrm{CHCl}_{3}\right) ;{ }^{1} \mathrm{H} \mathrm{NMR}\left(\mathrm{CDCl}_{3}, 500 \mathrm{MHz}\right): \delta 4.12(\mathrm{q}, J=7.5 \mathrm{~Hz}, 1 \mathrm{H})$, $1.65(\mathrm{~m}, 1 \mathrm{H}), 1.52(\mathrm{~m}, 1 \mathrm{H}), 1.46(\mathrm{~m}, 1 \mathrm{H}), 1.34(\mathrm{~m}, 1 \mathrm{H}), 1.24(\mathrm{~m}, 9 \mathrm{H}), 0.97(\mathrm{~m}, 1 \mathrm{H}), 0.90$ (m, 1H), 0.86 (m, 3H) ppm; ${ }^{13} \mathrm{C} \mathrm{NMR}\left(\mathrm{CDCl}_{3}, 400 \mathrm{MHz}\right): \delta$ 173.3, 60.4, 31.8, 29.5, 27.2, 22.8, 22.2, 18.4, 14.6, 14.2, 13.5 ppm; FTIR (film): 2922, 2856, 1724, 1438, 1398, 1380, 1170, $1000 \mathrm{~cm}^{-1}$; HRMS $\left(\mathrm{CI}^{+}\right)$calcd for $\mathrm{C}_{11} \mathrm{H}_{24} \mathrm{NO}_{2}\left(\mathrm{M}+\mathrm{NH}_{4}\right)^{+}$202.1807, found 202.1810.

$(1 S, 2 R, 3 S)$-Ethyl 2-(tri-n-butylstannyl)-3-pentylcyclopropanecarboxylate ${ }^{11}$

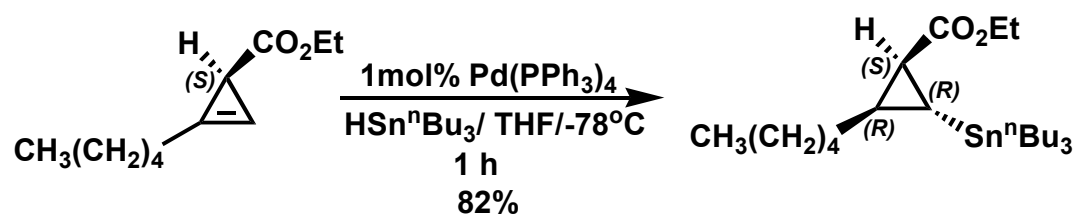

Palladium tetrakis-(triphenylphosphine) $(1 \mathrm{mg}, 0.0008 \mathrm{mmol})$ and tri- $n$-butyltin hydride $(0.025 \mathrm{~mL}, 0.093 \mathrm{mmol})$ were dissolved in THF $(1 \mathrm{~mL})$ and the solution was cooled to $-78{ }^{\circ} \mathrm{C}$. After 5 min, a solution of ethyl (1S)-2-n-pentyl-2-cyclopropenecarboxylate $(15 \mathrm{mg}, 0.082 \mathrm{mmol})$ in THF $(1 \mathrm{~mL})$ was added at $-78{ }^{\circ} \mathrm{C}$. The reaction mixture was warmed up to room temperature after $1 \mathrm{~h}$ and concentrated in vacuo. The residue was purified by column chromatography (20:1 hexanes-EtOAc) to give the desired product $(32 \mathrm{mg}, 82 \%)$ as colorless liquid: $[\alpha]^{23}{ }_{\mathrm{D}}=+109^{\circ}\left(\mathrm{c} 1.1, \mathrm{CHCl}_{3}\right) ;{ }^{1} \mathrm{H} \mathrm{NMR}$ $\left(\mathrm{CDCl}_{3}, 500 \mathrm{MHz}\right): \delta 4.12(\mathrm{~m}, 2 \mathrm{H}), 1.7-1.4(\mathrm{~m}, 9 \mathrm{H}), 1.35-1.15(\mathrm{~m}, 17 \mathrm{H}), 1.12(\mathrm{t}, J=4.5$ $\mathrm{Hz}, 1 \mathrm{H}), 0.98(\mathrm{dd}, J=4.5,6.5 \mathrm{~Hz}, 1 \mathrm{H}), 0.94-0.76(\mathrm{~m}, 16 \mathrm{H}) \mathrm{ppm} ;{ }^{13} \mathrm{C} \mathrm{NMR}\left(\mathrm{CDCl}_{3}, 500\right.$ MHz): $\delta$ 173.4, 60.3, 33.4, 32.2, 30.7, 29.3, 27.7, 22.9, 22.5, 21.3, 18.8, 14.6, 14.3, 13.9, 
$9.41 \mathrm{ppm}$; FTIR (film): 1723, 1457, 1377, $1155 \mathrm{~cm}^{-1}$; HRMS $\left(\mathrm{CI}^{+}\right)$calcd for $\mathrm{C}_{23} \mathrm{H}_{50} \mathrm{NO}_{2} \mathrm{Sn}\left(\mathrm{M}+\mathrm{NH}_{4}\right)^{+}$492.2863, found 492.2875.

Part 5. Synthesis of $(9 R, 10 S)$-Dihydrosterculic acid (12)

(R)-Ethyl 2-octylcycloprop-2-enecarboxylate (13)

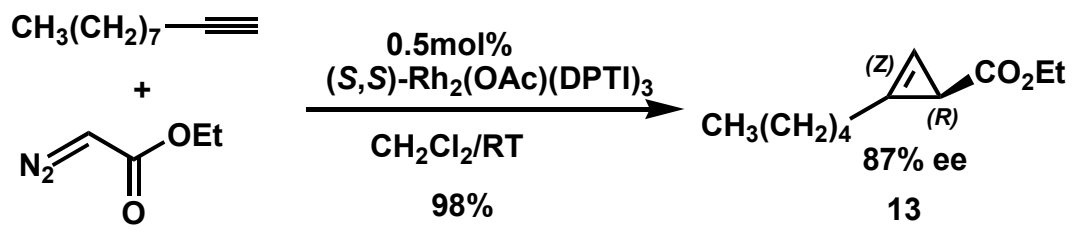

A solution of ethyl diazoacetate $(0.25 \mathrm{~mL}, 2.4 \mathrm{mmol})$ in dichloromethane $(2 \mathrm{~mL})$ was added slowly at room temperature to a mixture of $(S, S)-\mathrm{Rh}_{2}(\mathrm{OAc})(\mathrm{DPTI})_{3}(17 \mathrm{mg}, 0.012$ mmol) and 1-decyne $(1.0 \mathrm{~mL}, 5.7 \mathrm{mmol})$ in dichloromethane $(10 \mathrm{~mL})$ over $5 \mathrm{~h}$. After removal of volatiles, the residue was purified by column chromatography (40:1 hexanesEtOAc) to give the desired cyclopropene (13) $(0.52 \mathrm{~g}, 98 \%)$ as colorless oil. The enantiomeric excess was determined to be $87 \%$ by GC ( $\gamma$-TA column): $100{ }^{\circ} \mathrm{C} ; 25 \mathrm{psi} ; \mathrm{t}_{\mathrm{R}}$ $=80.8 \min ($ major $), \mathrm{t}_{\mathrm{R}}=75.9 \min ($ minor $) ;[\alpha]^{23}{ }_{\mathrm{D}}=-30^{\circ}\left(c\right.$ 1.55, $\left.\mathrm{CHCl}_{3}\right) ;{ }^{1} \mathrm{H} \mathrm{NMR}$ $\left(\mathrm{CDCl}_{3}, 500 \mathrm{MHz}\right): \delta 6.29$ (d, $\left.J=1.5 \mathrm{~Hz}, 1 \mathrm{H}\right), 4.09(\mathrm{~m}, 2 \mathrm{H}), 2.47$ (t, $\left.J=7.5 \mathrm{~Hz}, 2 \mathrm{H}\right)$, $2.09(\mathrm{~d}, J=1.5 \mathrm{~Hz}, 1 \mathrm{H}), 1.54$ (quintet, $J=7.5 \mathrm{~Hz}, 2 \mathrm{H}), 1.4-1.1(\mathrm{~m}, 15 \mathrm{H}), 0.84(\mathrm{t}, J=$ $7.5 \mathrm{~Hz}, 3 \mathrm{H}) \mathrm{ppm} ;{ }^{13} \mathrm{C} \mathrm{NMR}\left(\mathrm{CDCl}_{3}, 500 \mathrm{MHz}\right): \delta 176.8,115.8,94.1,60.3,32.0,29.4$, 29.4, 29.3, 26.8, 25.2, 22.8, 19.9, 14.614 .3 ppm; FTIR (film): 2921, 2856, 1720, 1458, 1364, 1336, 1246, 1176, $1029 \mathrm{~cm}^{-1}$; HRMS $\left(\mathrm{ES}^{+}\right)$: calcd for $\mathrm{C}_{14} \mathrm{H}_{25} \mathrm{O}_{2}(\mathrm{M}+\mathrm{H})^{+}$ 225.1854 found 225.1851 .

Recovery of the catalyst $(S, S)-1$ : after the complete elusion of cyclopropene 13, the blue band of 1 was eluted down with $\mathrm{CH}_{2} \mathrm{Cl}_{2}$ and concentrated in vacuo. The residue was 
purified with preparative thin-layer chromatography $\left(20: 1 \mathrm{C}_{6} \mathrm{H}_{6}-\mathrm{CH}_{3} \mathrm{CN}\right)$ to afford pure $\mathbf{1}$ as purple solid (11 mg, 65\% recovery). The recovered catalyst has the same ${ }^{1} \mathrm{H}$ NMR spectrum to that of the freshly prepared catalyst $\mathbf{1}$ and gave almost identical ee\% for this cyclopropenation reaction as the fresh catalyst $\mathbf{1}$.

$(1 R, 2 S)$-Ethyl 2-octylcyclopropanecarboxylate

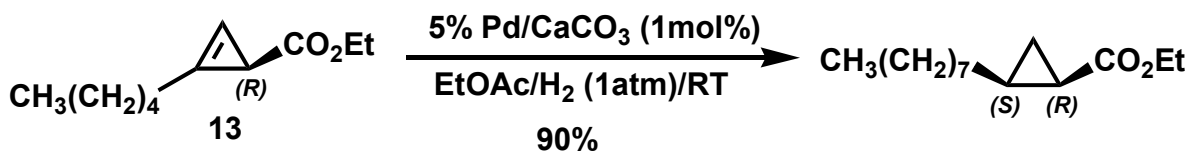

A suspension of cyclopropene $13(0.50 \mathrm{~g}, 2.2 \mathrm{mmol})$ and 5\% $\mathrm{Pd}$ on $\mathrm{CaCO}_{3}(70 \mathrm{mg}$, $0.022 \mathrm{mmol})$ in ethyl acetate $(20 \mathrm{~mL})$ was vigorously stirred under hydrogen atmosphere. The reaction progress was monitored by TLC analysis until the complete consumption of starting material. The reaction mixture was filtered through a short plug of Celite, and concentrated in vacuo. The residue was purified by column chromatography (40:1 hexanes-EtOAc) to give the desired cyclopropane (450 mg, 90\%) as colorless liquid: $[\alpha]^{23}{ }_{\mathrm{D}}=-28^{\mathrm{o}}\left(c\right.$ 5.0, $\left.\mathrm{CHCl}_{3}\right) ;{ }^{1} \mathrm{H} \mathrm{NMR}\left(\mathrm{CDCl}_{3}, 500 \mathrm{MHz}\right): \delta 4.20(\mathrm{q}, J=7.5 \mathrm{~Hz}, 2 \mathrm{H})$, $1.65(\mathrm{~m}, 1 \mathrm{H}), 1.52(\mathrm{~m}, 1 \mathrm{H}), 1.45(\mathrm{~m}, 1 \mathrm{H}), 1.33(\mathrm{~m}, 1 \mathrm{H}), 1.24(\mathrm{~m}, 15 \mathrm{H}), 0.98(\mathrm{~m}, 1 \mathrm{H})$, $0.89(\mathrm{~m}, 1 \mathrm{H}), 0.85(\mathrm{t}, J=7.5 \mathrm{~Hz}, 3 \mathrm{H}) \mathrm{ppm} ;{ }^{13} \mathrm{C} \mathrm{NMR}\left(\mathrm{CDCl}_{3}, 500 \mathrm{MHz}\right): \delta 173.2,60.4$, $32.1,29.9,29.8,29.5,29.5,27.2,22.9,22.2,18.4,14.6,14.3,13.5$ ppm; FTIR (film): 2925, 2859, 1724, 1438, 1399, 1382, 1170, $1096 \mathrm{~cm}^{-1}$; HRMS $\left(\mathrm{CI}^{+}\right)$calcd for $\mathrm{C}_{14} \mathrm{H}_{30} \mathrm{NO}_{2}$ $\left(\mathrm{M}+\mathrm{NH}_{4}\right)^{+}$244.2277, found 244.2276.

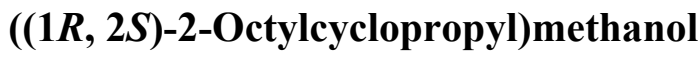

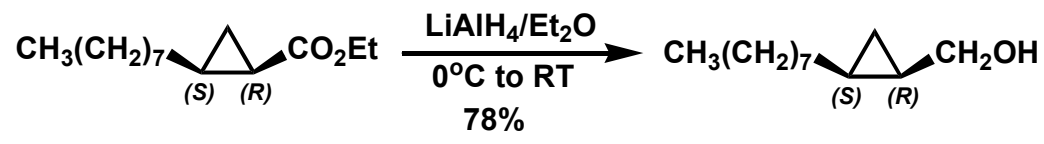


Lithium aluminum hydride $(27 \mathrm{mg}, 0.71 \mathrm{mmol})$ was added in one portion at $0{ }^{\circ} \mathrm{C}$ to a solution of cyclopropyl ester $(80 \mathrm{mg}, 0.35 \mathrm{mmol})$ in ether $(2 \mathrm{~mL})$. The suspension was warmed to room temperature and stirred for $1 \mathrm{~h}$. The reaction mixture was treated with saturated $\mathrm{Na}_{2} \mathrm{SO}_{4}$ solution $(20 \mathrm{~mL})$ and extracted with ether $(20 \mathrm{~mL}$ x 2$)$. The extracted organic solution was washed with brine $(20 \mathrm{~mL})$ and concentrated in vacuo. The residue was purified by column chromatography (6:1 hexanes-EtOAc) to give the desired alcohol (51 mg, 78\%): $[\alpha]^{23}{ }_{\mathrm{D}}=+19^{\circ}\left(\mathrm{c} 3.25, \mathrm{CHCl}_{3}\right) ;{ }^{1} \mathrm{H} \mathrm{NMR}\left(\mathrm{CDCl}_{3}, 500 \mathrm{MHz}\right): \delta 3.65$ (dd, $J$ $=7.5,11 \mathrm{~Hz}, 1 \mathrm{H}), 3.56(\mathrm{dd}, J=7.5,11 \mathrm{~Hz}, 1 \mathrm{H}), 1.51(\mathrm{br} \mathrm{s}, 1 \mathrm{H}), 1.50-1.15(\mathrm{~m}, 14 \mathrm{H}), 1.10$ $(\mathrm{m}, 1 \mathrm{H}), 0.88(\mathrm{~m}, 4 \mathrm{H}), 0.69(\mathrm{dt}, J=5.0,8.0 \mathrm{~Hz}, 1 \mathrm{H}),-0.04(\mathrm{q}, J=5.0 \mathrm{~Hz}, 1 \mathrm{H}) \mathrm{ppm} ;{ }^{13} \mathrm{C}$ NMR ( $\left.\mathrm{CDCl}_{3}, 400 \mathrm{MHz}\right): \delta$ 63.6, 32.2, 30.4, 29.9, 29.8, 29.6, 28.8, 22.9, 18.4, 16.4, 14.4, 9.7 ppm; FTIR (film): 3323 (br), 2921, 2850, 1467, $1030 \mathrm{~cm}^{-1}$; $\mathrm{HRMS}\left(\mathrm{CI}^{+}\right)$calcd for $\mathrm{C}_{12} \mathrm{H}_{28} \mathrm{NO}\left(\mathrm{M}+\mathrm{NH}_{4}\right)^{+}$202.2171, found 202.2176.

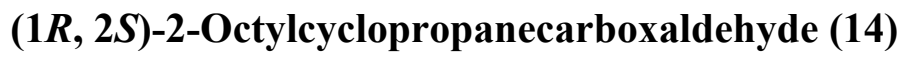

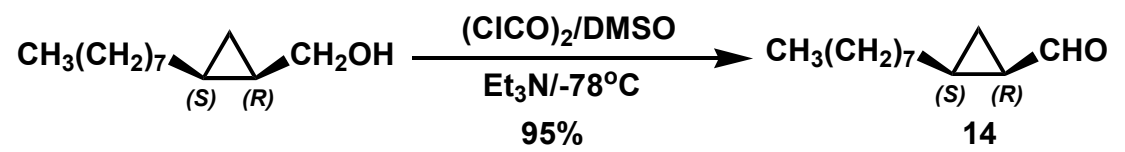

A solution of DMSO $(0.118 \mathrm{~mL}, 1.66 \mathrm{mmol})$ in dichloromethane $(1 \mathrm{~mL})$ was added dropwise at $-78{ }^{\circ} \mathrm{C}$ to a solution of oxalyl chloride $(0.072 \mathrm{~mL}, 0.83 \mathrm{mmol})$ in dichloromethane $(1.5 \mathrm{~mL})$. After $10 \mathrm{~min}$, a solution of alcohol $(51 \mathrm{mg}, 0.28 \mathrm{mmol})$ in dichloromethane $(1 \mathrm{~mL})$ was added and the resulted mixture was stirred for $30 \mathrm{~min}$ before $\mathrm{Et}_{3} \mathrm{~N}(0.347 \mathrm{~mL}, 2.49 \mathrm{mmol})$ was slowly added at $-78{ }^{\circ} \mathrm{C}$. The reaction mixture was warmed to room temperature 15 min later and treated with saturated $\mathrm{NH}_{4} \mathrm{Cl}$ solution $(10 \mathrm{~mL})$. The resulting mixture was then extracted with ether $(15 \mathrm{~mL} \times 2)$, washed with brine $(15 \mathrm{~mL})$, dried over $\mathrm{MgSO}_{4}$ and concentrated in vacuo. The residue was purified 
with column chromatography (20:1 hexanes-EtOAc) to give aldehyde 14 (48 mg, 95\%) as colorless liquid: $[\alpha]^{23}{ }_{\mathrm{D}}=+12^{\circ}\left(c 1.0, \mathrm{CHCl}_{3}\right) ;{ }^{1} \mathrm{H} \mathrm{NMR}\left(\mathrm{CDCl}_{3}, 500 \mathrm{MHz}\right): \delta 9.34(\mathrm{~d}$, $J=5.5 \mathrm{~Hz}, 1 \mathrm{H}), 1.85(\mathrm{~m}, 1 \mathrm{H}), 1.59(\mathrm{~m}, 1 \mathrm{H}), 1.48(\mathrm{~m}, 2 \mathrm{H}), 1.38(\mathrm{~m}, 1 \mathrm{H}), 1.34-1.12(\mathrm{~m}$, $13 \mathrm{H}), 0.87(\mathrm{t}, J=7.0 \mathrm{~Hz}, 3 \mathrm{H}) \mathrm{ppm} ;{ }^{13} \mathrm{C} \mathrm{NMR}\left(\mathrm{CDCl}_{3}, 500 \mathrm{MHz}\right): \delta 202.1,32.1,30.2$, 29.7, 29.5, 28.5, 28.0, 25.0, 22.9, 15.0, 14.3 ppm; FTIR (film): 2920, 2853, 1704, 1462, 1452, 1366, $1177 \mathrm{~cm}^{-1}$; HRMS $\left(\mathrm{CI}^{+}\right)$calcd for $\mathrm{C}_{12} \mathrm{H}_{26} \mathrm{NO}\left(\mathrm{M}+\mathrm{NH}_{4}\right)^{+} 200.2114$, found 202.2114 .

9-((1R, 2S)-2-Octylcyclopropyl)non-8-enoic acid (15)

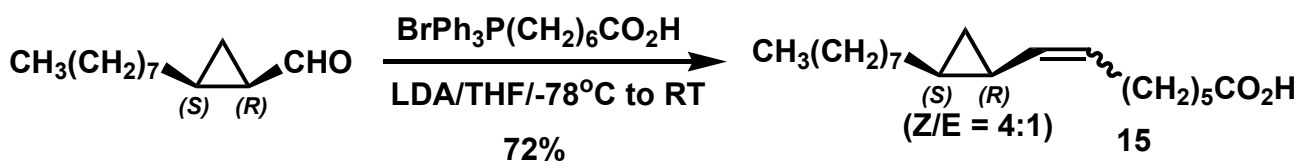

${ }^{\mathrm{n}} \mathrm{BuLi}(2.87 \mathrm{M}, 0.276 \mathrm{~mL})$ was added to a solution of $\mathrm{i}-\mathrm{Pr}_{2} \mathrm{NH}(0.112 \mathrm{~mL}, 0.791$ mmol) in THF $(2 \mathrm{~mL})$ at $-78{ }^{\circ} \mathrm{C}$ and stirred at this temperature for $0.5 \mathrm{~h}$. Then $\mathrm{BrPh}_{3} \mathrm{P}\left(\mathrm{CH}_{2}\right)_{6} \mathrm{CO}_{2} \mathrm{H}^{12}(186 \mathrm{mg}, 0.396 \mathrm{mmol})$ was added in one portion at $-78{ }^{\circ} \mathrm{C}$. The mixture was allowed to warm to room temperature and stirred for $2 \mathrm{~h}$ when the color of the reaction mixture turned orange red. A solution of aldehyde $14(48 \mathrm{mg}, 0.26 \mathrm{mmol})$ in THF $(1 \mathrm{~mL})$ was introduced dropwise via syringe at room temperature. The reaction mixture was stirred overnight before the treatment with $1 \mathrm{~N} \mathrm{HCl}$ solution $(15 \mathrm{~mL})$. The resulting mixture was extracted with ether $(15 \mathrm{~mL} \times 2)$, washed with brine $(15 \mathrm{~mL})$, dried with $\mathrm{MgSO}_{4}$, and concentrated in vacuo. The residue was purified by column chromatography (5:1 hexanes-EtOAc) to provide $15(58 \mathrm{mg}, 72 \%$, cis: trans $=\sim 4: 1$ by ${ }^{1} \mathrm{H}$ NMR integration) as colorless liquid: $[\alpha]^{23}{ }_{\mathrm{D}}=+40^{\circ}\left(c 1.2, \mathrm{CHCl}_{3}\right) .{ }^{1} \mathrm{H}$ NMR for the cis isomer $\left(\mathrm{CDCl}_{3}, 400 \mathrm{MHz}\right): \delta 9.49(\mathrm{br} \mathrm{s}), 5.38(\mathrm{dt}, J=10.8,6.8 \mathrm{~Hz}, 1 \mathrm{H}), 5.04(\mathrm{dd}, J=$ 9.2, 10.8Hz, 1H), $2.32(\mathrm{~m}, 3 \mathrm{H}), 2.15(\mathrm{q}, J=6.4 \mathrm{~Hz}, 2 \mathrm{H}), 1.64(\mathrm{~m}, 2 \mathrm{H}), 1.54(\mathrm{~m}, 1 \mathrm{H})$, 
1.43-1.20 (m, 18H), $0.89(\mathrm{~m}, 4 \mathrm{H}), 0.09(\mathrm{~m}, 1 \mathrm{H}) \mathrm{ppm} ;{ }^{13} \mathrm{C}$ NMR for the cis isomer $\left(\mathrm{CDCl}_{3}, 400 \mathrm{MHz}\right): \delta 179.7,129.8,129.7,31.9,29.7,29.6,29.5,29.4,29.4,29.3,28.7$, 27.3, 24.7, 22.7, 18.5, 14.2, 14.1, 13.9 ppm; FTIR (film): 2921, 2853, 1707, 1462, 1452, 1413, 1278, 1225, $1027 \mathrm{~cm}^{-1}$; HRMS $\left(\mathrm{CI}^{+}\right)$calcd for $\mathrm{C}_{19} \mathrm{H}_{38} \mathrm{NO}_{2}\left(\mathrm{M}+\mathrm{NH}_{4}\right)^{+}$312.2903, found 312.2893

$(9 R, 10 S)$-Dihydrosterculic acid (12)

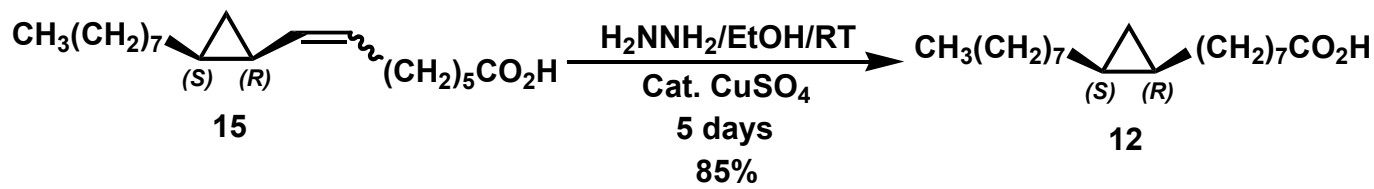

An isomeric mixture of $15(0.70 \mathrm{~g}, 2.3 \mathrm{mmol})$ was dissolved in 95\% ethanol (40 mL) and anhydrous hydrazine $(10 \mathrm{~mL})$. A saturated aqueous $\mathrm{CuSO}_{4}$ solution $(1 \mathrm{~mL})$ was added in five equal portions once every day. The mixture was vigorously stirred at room temperature for 5 days when ${ }^{1} \mathrm{H}$ NMR analysis of an aliquot showed the complete consumption of the starting material 15. The reaction mixture was acidified with concentrated $\mathrm{HCl}$ solution and extracted with ether $(15 \mathrm{~mL} \times 4)$ and the combined organic layer was washed with $2 \mathrm{~N} \mathrm{HCl}$ solution $(30 \mathrm{~mL})$ and brine $(30 \mathrm{~mL})$, dried over $\mathrm{MgSO}_{4}$ and concentrated in vacuo. The residue was purified by column chromatography (5:1 hexanes-EtOAc) to afford an almost colorless solid, which was crystallized from petroleum ether at $-50{ }^{\circ} \mathrm{C}$ to give dihydrosterculic acid (12) $(0.60 \mathrm{~g}, 85 \%)$ as colorless solid, mp 29-30 ${ }^{\circ} \mathrm{C}^{13} ;[\alpha]^{23} \mathrm{D}=+0.92^{\circ}\left(c\right.$ 14, $\left.\mathrm{CHCl}_{3}\right) ;{ }^{1} \mathrm{H} \mathrm{NMR}\left(\mathrm{CDCl}_{3}, 500 \mathrm{MHz}\right): \delta 9.8$ (br s, 1H), $2.35(\mathrm{t}, J=7.5 \mathrm{~Hz}, 2 \mathrm{H}), 1.64$ (quintet, $J=2.0 \mathrm{~Hz}, 2 \mathrm{H}), 1.43-1.20(\mathrm{~m}, 22 \mathrm{H})$, $1.14(\mathrm{~m}, 2 \mathrm{H}), 0.89(\mathrm{t}, J=8.0 \mathrm{~Hz}, 3 \mathrm{H}), 0.65(\mathrm{br} \mathrm{s}, 2 \mathrm{H}), 0.56(\mathrm{dt}, J=4.0,8.0 \mathrm{~Hz}, 1 \mathrm{H}),-0.33$ $(\mathrm{q}, J=4.0 \mathrm{~Hz}, 1 \mathrm{H}) \mathrm{ppm} ;{ }^{13} \mathrm{C} \mathrm{NMR}\left(\mathrm{CDCl}_{3}, 500 \mathrm{MHz}\right): \delta 180.0,31.9,31.6,30.2,30.1$, 29.7, 29.4, 29.4, 29.3, 29.1, 28.7, 28.6, 24.7, 22.7, 22.6, 15.8, 15.7, 14.1, 10.9 ppm; FTIR 
(film): 2917, 2850, 1708, 1216, $934 \mathrm{~cm}^{-1}$; HRMS $\left(\mathrm{CI}^{+}\right)$calcd for $\mathrm{C}_{19} \mathrm{H}_{40} \mathrm{NO}_{2}\left(\mathrm{M}+\mathrm{NH}_{4}\right)^{+}$ 314.3059, found 314.3051 .

Part 6. ORTEP Presentations of Catalysts $\mathbf{1 ,} 2$ and $\mathbf{1 0}{ }^{14,15}$

Figure 1. ORTEP presentation of $(R, R)-\mathrm{Rh}_{2}(\mathrm{OAc})(\mathrm{DPTI})_{3}(\mathbf{1})$

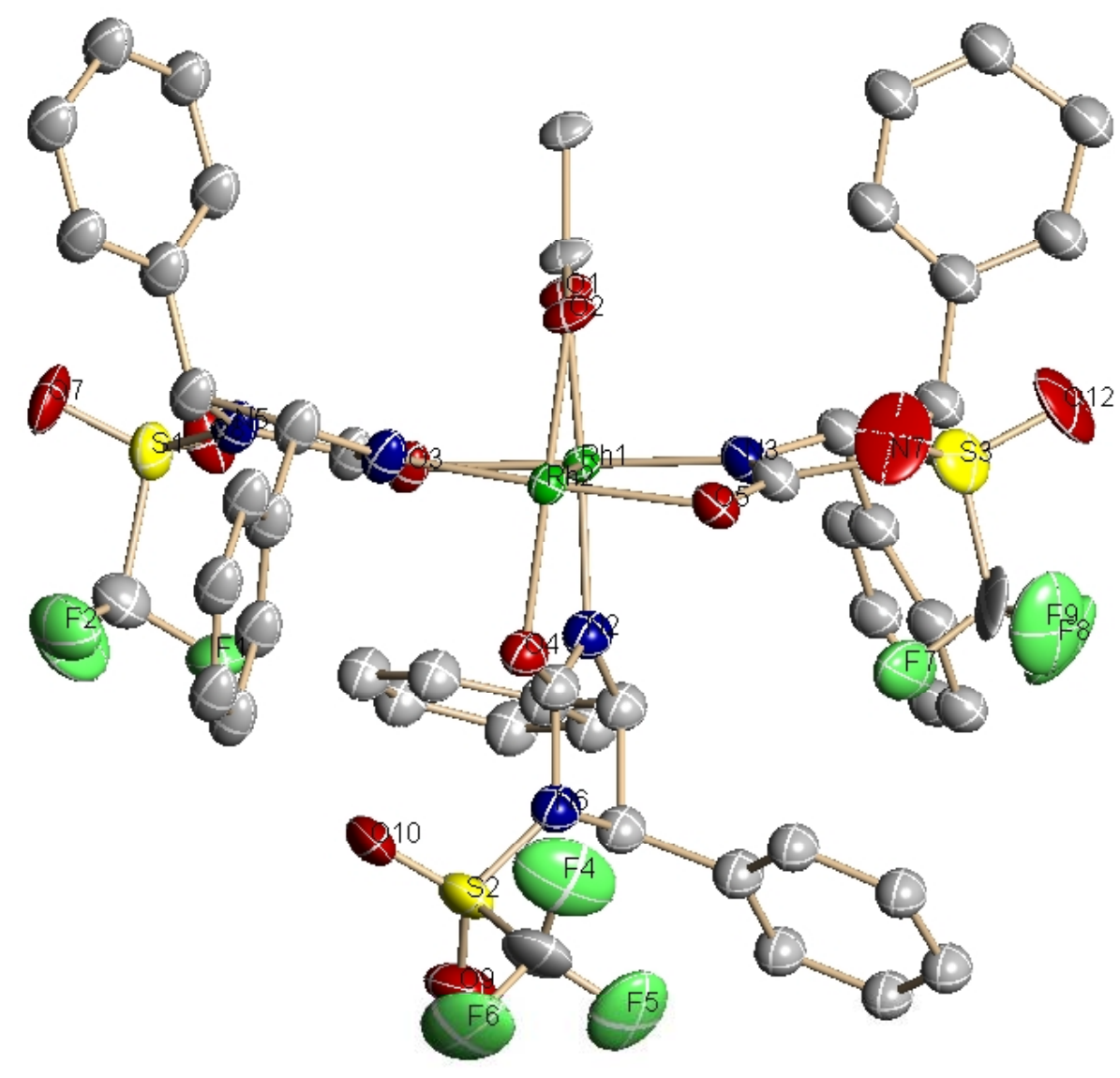


S 26

Figure 2. ORTEP presentation of $(R, R)-\mathrm{Rh}_{2}(\mathrm{DPTI})_{4}(\mathbf{2})$

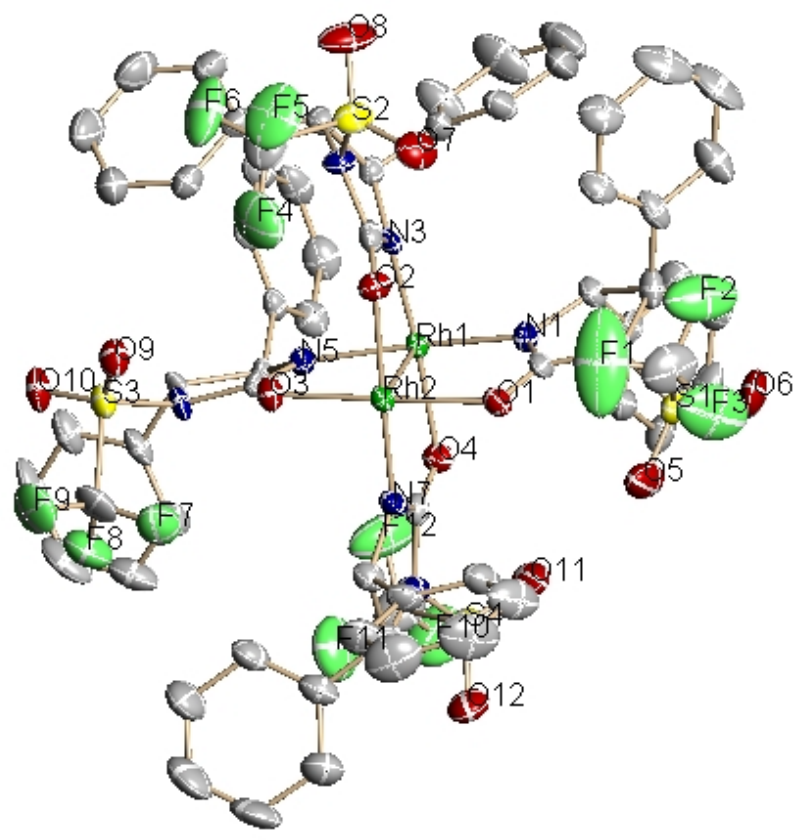

Figure 3. ORTEP presentation of $(M)-(R, R)-c i s-(\mathrm{N}, \mathrm{O})-\mathrm{Rh}_{2}(\mathrm{Piv})_{2}(\mathrm{DPTI})_{2}(\mathbf{1 0})$

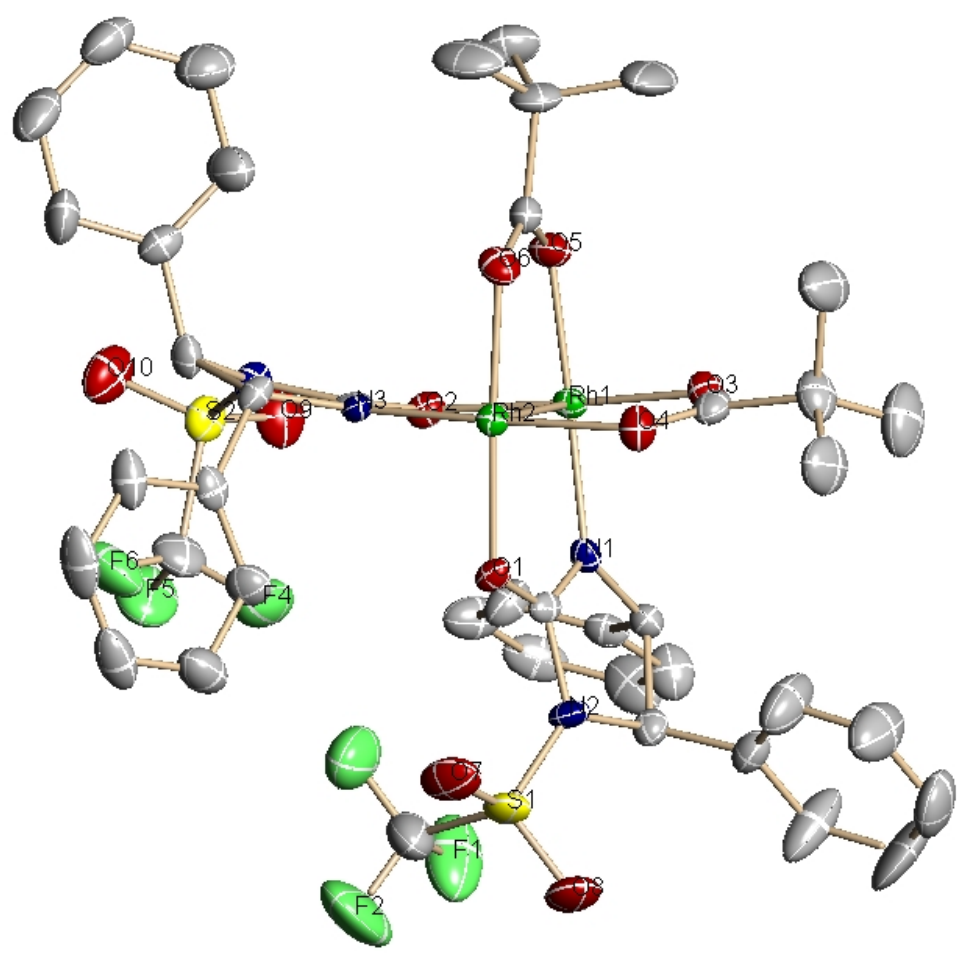




\section{References}

1. Knolker, H-J.; Braxmeier, T. Tetrahedron Lett. 1998, 39, 9407-9410.

2. For a report of the absolute configuration of the corresponding methyl esters, see: Doyle, M. P.; Protopopova, M.; Müller, P.; Ene, D.; Shapiro, E. A. J. Am. Chem. Soc. 1994, 116, 8492-8498.

3. Müller, P.; Imogaï, H. Tetrahedron:Asymmetry 1998, 9, 4419-4428.

4. Watanabe, N.; Matsuda, H.; Kuribayashi, H. and Hashimoto, S. Heterocycles 1996, 42, 537-542.

5. Sanders, C. J.; Gillespie, K. M.; Scott, P. Tetrahedron: Asymmetry 2001, 12, 1055-1061.

6. Saito, H.; Oishi, H.; Kitagaki, S.; Nakamura, S.; Anada, M.; Hashimoto, S. Org. Lett. 2002, 4, 3887-3890.

7. For leading references of cycloaddition reactions of cyclopropenes with cyclopentadiene, see: (a) Wiberg, K. B.; Bartley, W. J. J. Am. Chem. Soc. 1960, 82, 6375-6378. (b) Paulini, K.; Reissig, J.-U. Synlett 1992, 6, 505-507. (c) Binger, P.; Wedemann, P.; Goddard, R.; Brinker, U. H. J. Org. Chem. 1996, 61, 64626464.

8. For a discussion of $\mathrm{LiAlH}_{4}$ reduction of cyclopropenyl carboxylates, see: (a) Vincens, M.; Dumont, C.; Vidal, M. Tetrahedron 1983, 39, 4281-4289. (b) Zohor, E.; Marek, I. Org. Lett. 2004, 6, 341-343.

9. For a reference of DIBAL reduction of cyclopropenyl carboxylates and the useful transformations with the resulting cyclopropenyl alcohols, see: Liao, L.-a.; Fox, J. M. J. Am. Chem. Soc. 2002, 124, 14322-14323. 
10. For a reference of $\mathrm{Pd} / \mathrm{C}$ hydrogenation of cyclopropenes, see: Imogaï, H.; Bernardinelli, G.; Gränicher, C; Moran, M; Rossier, J.-C.; Müller, P. Helv. Chim. Acta 1998, 81, 1754-1764.

11. For a recent reference of hydrostannation of cyclopropenes, see: Rubina, M.; Rubin, M.; Gevorgyan, V. J. Am. Chem. Soc. 2002, 124, 11566-11567.

12. Kato, K.; Ohkawa, S.; Terao, S.; Terashita, Z.; Nishikawa, K. J. Med. Chem. 1985, 28, 287-294.

13. DL-Dihydrosterculic acid was reported to have a melting point of $34-37^{\circ} \mathrm{C}$ : Simmons, H. E.; Smith, R. D. J. Am. Chem. Soc. 1959, 81, 4256-4264; See Grossert, J. S.; Ratnayake, W. M. N.; Swee, T. Can . J. Chem. 1981, 59, 2617 for its ${ }^{1} \mathrm{H}$ NMR data in $\mathrm{CDCl}_{3}: \delta-0.3(1 \mathrm{H}, \mathrm{m}), 0.6(3 \mathrm{H}, \mathrm{m}), 0.9(3 \mathrm{H}, \mathrm{m}), 1.3(26 \mathrm{H}$, $\mathrm{m}), 2.2-2.3(2 \mathrm{H}, \mathrm{m})$, and $9.7-10.0(1 \mathrm{H}, \mathrm{br} \mathrm{s})$.

14. We are grateful to Dr. Richard J. Staples at X-ray crystallographic laboratory of Harvard Department of Chemistry and Chemical Biology for the determination of the structures of catalysts $\mathbf{1}, \mathbf{2}$ and $\mathbf{1 0 .}$

15. The axially coordinated molecules and solvent molecules were removed from the ORTEP presentations of catalysts $\mathbf{1}, \mathbf{2}$ and $\mathbf{1 0}$ for better viewing. 